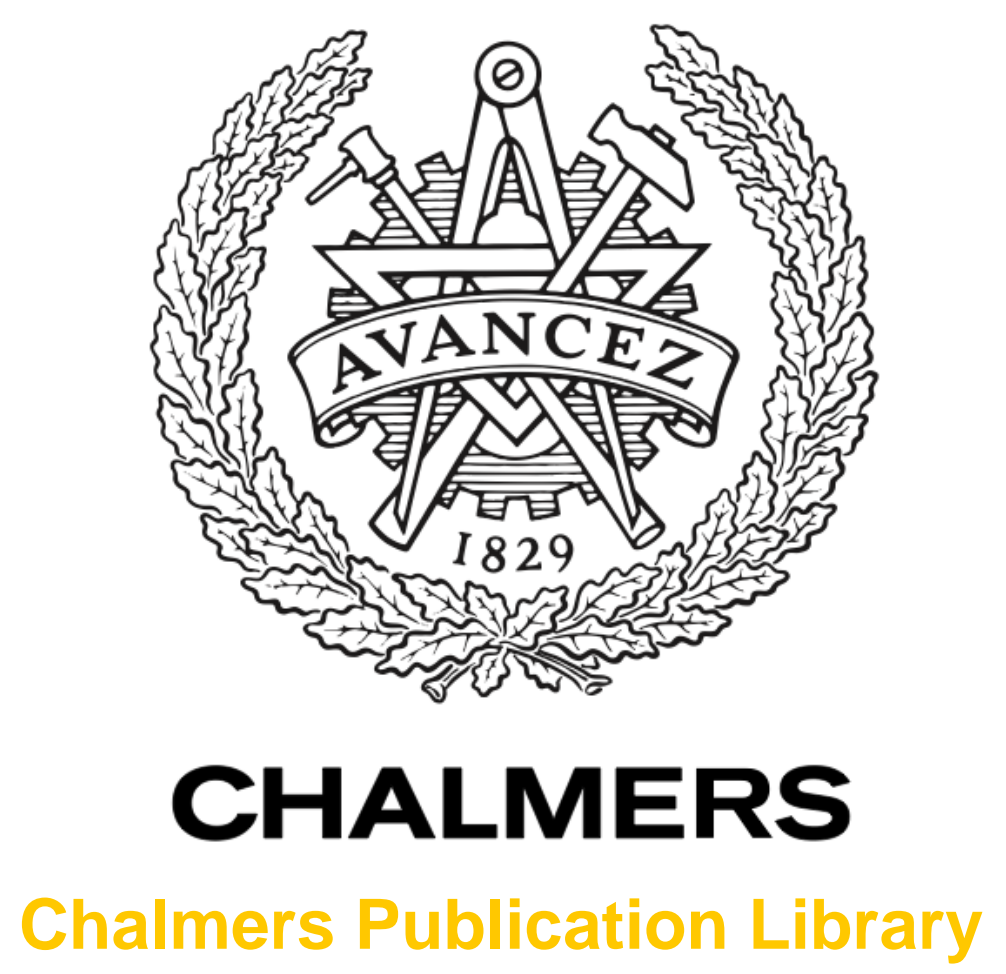

Plasma response based RMP coil geometry optimization for an ITER plasma

This document has been downloaded from Chalmers Publication Library (CPL). It is the author's version of a work that was accepted for publication in:

Plasma Physics and Controlled Fusion (ISSN: 07413335)

Citation for the published paper:

Zhou, L. ; Liu, Y. ; Liu, Y. et al. (2016) "Plasma response based RMP coil geometry optimization for an ITER plasma". Plasma Physics and Controlled Fusion, vol. 58(11), pp. 115003.

http://dx.doi.org/10.1088/0741-3335/58/11/115003

Downloaded from: http://publications.lib.chalmers.se/publication/246214

Notice: Changes introduced as a result of publishing processes such as copy-editing and formatting may not be reflected in this document. For a definitive version of this work, please refer to the published source. Please note that access to the published version might require a subscription. 


\title{
Plasma response based RMP coil geometry optimization for an ITER plasma
}

\author{
Lina Zhou ${ }^{1}$, Yueqiang $\mathrm{Liu}^{2,3,4}$, Yue $\mathrm{Liu}^{1}$ and $\mathrm{Xu} \mathrm{Yang}{ }^{1}$ \\ ${ }^{1}$ Key Laboratory of Materials Modification by Laser, Ion, and Electron Beams (Ministry of \\ Education), School of Physics and Optoelectronic Technology, Dalian University of Technology, \\ Dalian 116024, China \\ ${ }^{2}$ CCFE, Culham Science Centre, Abingdon, OX14 3DB, UK \\ ${ }^{3}$ Southwestern Institute of Physics, PO Box 432, Chengdu 610041, China \\ ${ }^{4}$ Department of Earth and Space Science, Chalmers University of Technology, SE-412 96 \\ Gothenburg, Sweden \\ E-mails of corresponding authors: yueqiang.liu@ukaea.uk; liuyue@dlut.edu.cn
}

\begin{abstract}
Based on an ITER 15MA $\mathrm{Q}=10$ inductive scenario, systematic numerical investigation is carried out, in order to understand the effect of varying the geometry of the magnetic coils, used for controlling the edge localized modes in tokamaks, on the plasma response to the resonant magnetic perturbation (RMP) fields produced by these coils. Toroidal computations show that both of the plasma response based figures of merit - one is the pitch resonant radial field component near the plasma edge and the other is the plasma displacement near the X-point of the separatrix - consistently yield the same prediction for the optimal coil geometry. With couple of exceptions, the presently designed poloidal location of the ITER upper and lower rows of RMP coils is close to the optimum, according to the plasma response based criteria. This holds for different coil current configurations with $n=2,3,4$, as well as different coil phasing between the upper and lower rows. The coils poloidal width from the present design, on the other hand, is sub-optimal for the upper and lower rows. Modelling also finds that the plasma response amplitude sharply decreases by moving the middle row RMP coils of ITER from the designed radial location (just inside the inner vacuum vessel) outwards (outside the outer vacuum vessel). The decay rate is sensitively affected by the middle row coils' poloidal coverage for low- $n(n=1,2)$ RMP fields, but not for high- $n(n=4)$ fields.
\end{abstract}

Keywords: RMP response, geometry optimization, ITER

\section{Introduction}

The edge localized modes (ELMs), appearing as bursting plasma instabilities, are a ubiquitous feature of tokamak H-mode discharges [1,2]. The large scale, low frequency type-I ELMs may pose serious risks to machine components in future large fusion devices such as ITER 
[3]. As an effective technique affecting the behavior of ELMs, the externally applied resonant magnetic perturbation (RMP) fields can either suppress or mitigate ELMs, as has been shown in experiments [4-8].

During recent years, significant efforts have been devoted to modelling various aspects of the ELM control with RMP fields [9-25]. It has now been well established that the toroidal and poloidal spectra of the applied 3D fields play crucial role in controlling the ELMs behavior $[16,21,25,26]$, as well as introducing side effects on the plasma density and toroidal flow [16,27]. Whilst the choice of the toroidal mode number $(n)$ for the 3D perturbation is determined by the number of coils along the toroidal angle of the torus, that can be afforded on a given tokamak device, the choice of the poloidal spectrum is not straightforward. The poloidal spectrum of the applied field perturbation depends both on the geometry of the coils and, in case of more than one rows of coils along the poloidal angle, the relative toroidal phasing of the coil currents in each row. The geometry of the coils includes the radial and poloidal location of the coils, the coverage (or extension) of each coil along the poloidal angle. This assumes that the shape of each coil is fixed, which is often the window-frame like, as has been designed in majority of the present day devices, as well as in ITER.

For a strongly shaped toroidal plasma, which is often the case with divertor configurations, quantitative information of the poloidal spectrum of the applied field can only be computationally obtained. Moreover, the plasma response often significantly modifies the poloidal spectrum of the applied field [11], in particular if the applied field has low toroidal mode number $n$ (typically between 1-6), which is usually the case in experiments. Therefore, toroidal computation of the RMP spectrum, including the plasma response, is one of the key aspects of the ELM control modelling. In particular, optimization of the poloidal spectrum of the RMP fields including the plasma response, by optimizing the coil geometry, is a very important, but far less explored topic.

This work offers a systematic investigation on the RMP coil geometry optimization, including the plasma response modelled by the MARS-F code [28]. We performed the study for an ITER plasma from the $15 \mathrm{MA} \mathrm{Q}=10$ inductive scenario [29] as an example. The same approach can be applied for the coil design optimization in any other future devices, such as the HL-2M tokamak currently under construction in China. The present ELM control coil design in ITER involves three rows of coils, referred to as the upper, middle, and lower rows in this work. Each row has 9 coils distributed along the toroidal angle [30]. These coils are all located just inside the inner vacuum vessel but beyond the blanket modules of ITER. In this study, we shall investigate two independent cases. In one case, we only use the upper and lower rows for the ELM control, and we shall vary the poloidal location, the poloidal coverage of the coils, as well as the coil phasing between the upper and lower row coil currents. In the other case, assuming that only the middle row of coils are utilized for the ELM control, we shall vary the poloidal coverage as well as the radial location of the coils.

In order to determine the "optimal" coil geometry, we need to define certain criteria (figures of merit). In this work, we consider two types of criteria. One is based on the amplitude of the 
MARS-F computed pitch resonant radial magnetic field near the plasma edge. The other is based on the computed plasma surface displacement. The choice of these criteria is motivated by the recent work, showing good correlations between the edge resonant radial field harmonic (including the plasma response) and the plasma displacement near the X-point (for divertor plasma configurations), and the achieved ELM mitigation in RMP experiments in MAST [16], ASDEX Upgrade [25], DIII-D [31] and EAST [32].

The paper is organized as follows. In section 2, we introduce the plasma equilibrium and coil configurations for the ITER example. Section 3 briefly describes the linear plasma response model in MARS-F. Section 4 presents the computational results involving systematic scan of parameters describing the RMP coil geometry. Section 5 summarizes the work.

\section{Plasma equilibrium and coil configurations}

We consider an ITER equilibrium from the 15MA baseline scenario obtained from the CORSICA transport simulation [29,33]. The same equilibrium has recently been considered in another work studying effects of the RMP fields on the plasma flow [23]. Figure 1 shows the plasma boundary shape and the modelled double wall shapes. The geometry of the RMP control coils, which we shall vary in this work, is also sketched. For each row of the coils, we define the poloidal angle of the center of the coil, $\theta_{c}$, as the poloidal location. The poloidal coverage of each coil is defined as $\Delta \theta$. In this work, except for cases reporting the computational results using the ITER designed coil geometry, we shall assume that the upper and the lower rows of coils have the same poloidal coverage (or coil's poloidal width, as will be referred to further-on). For the upper and lower rows, we fix the radial location of the coils to the ITER design, as shown in Fig. 1. For the middle row, however, we shall also vary the radial location, $r_{\mathrm{c}}$, of the coils. Sketched in Fig. 1 is an example where the middle row is located outside the ITER vacuum vessel.

We point out that Fig. 1 here does not show the coil geometry from the ITER design. Note that both $\theta_{c}$ and $\Delta \theta$ are defined in this work using the geometric poloidal angle. For the RMP coil design [34], adopted in ITER and also assumed in [23], $\theta_{c}^{U}=39.0^{\circ}, \Delta \theta^{U}=15.5^{\circ}$, for upper row coils; $\theta_{c}^{M}=0^{o}, \Delta \theta^{M}=35.9^{\circ}$ for middle row coils, and $\theta_{c}^{L}=-41.1^{\circ}, \Delta \theta^{L}=14.1^{\circ}$ for lower row coils. 


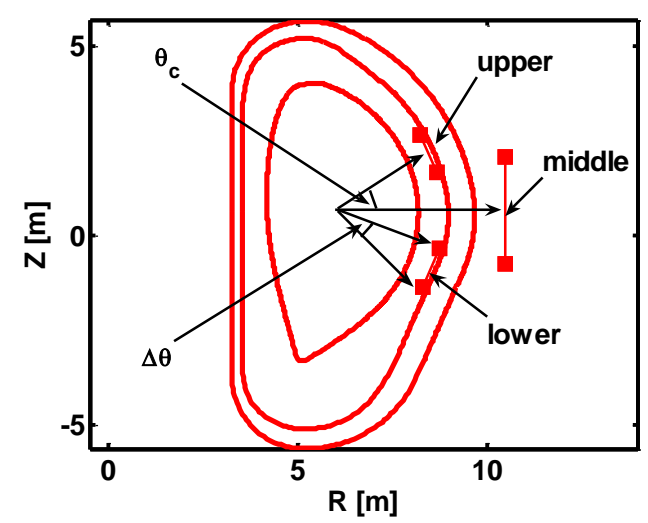

Figure 1. The location of the center and the coverage of the RMP coils, along the geometric poloidal angle, are defined via $\theta_{c}$ and $\Delta \theta$, respectively, for both upper and lower rows of coils. The radial location of the middle row coils is varied in the modelling. Plotted are also the plasma boundary shape and the (simplified) double wall shapes for the ITER 15MA baseline scenario.

Figure 2 shows the key equilibrium radial profiles obtained by CORSICA for this ITER plasma. The safety factor has on-axis value $q_{0}$ slightly below $1, q_{95}=3.18$ at the $95 \%$ equilibrium poloidal flux surface. The plasma pressure is normalized by $P_{0} \equiv B_{0}^{2} / \mu_{0}$, with $B_{0}=5.3 \mathrm{~T}, \mu_{0}=4 \pi \times 10^{-7} \mathrm{Hm}^{-1}$. The toroidal plasma current density $J_{\varphi}$ is normalized by $J_{0} \equiv B_{0} /\left(\mu_{0} R_{0}\right)$, with $R_{0}=6.2 \mathrm{~m}$. The plasma density is normalized to unity at the magnetic axis.
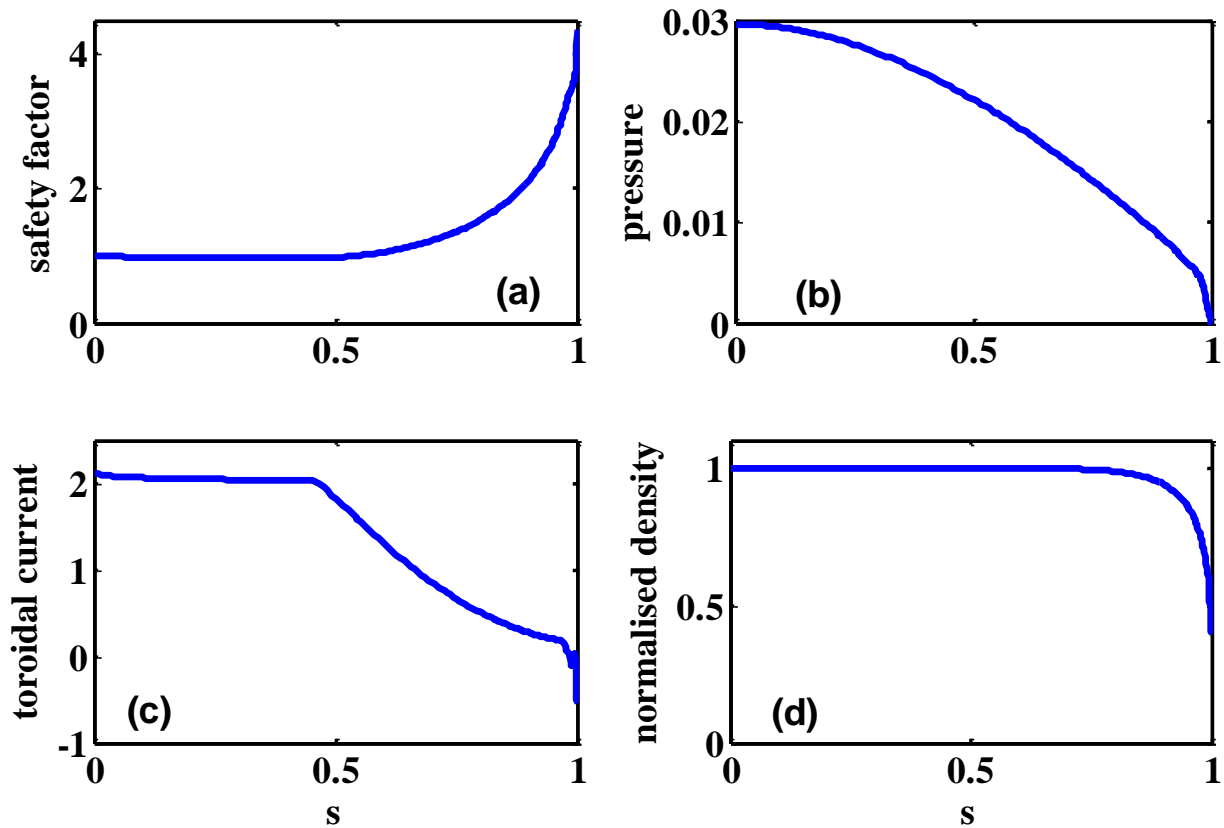

Figure 2. Equilibrium radial profiles of $(a)$ the safety factor, $(b)$ the plasma pressure normalized by $B_{0}^{2} / \mu_{0},(c)$ the surface averaged toroidal current density normalized by $B_{0} / \mu_{0} R_{0}$, and $(d)$ the plasma density normalized to unity on the magnetic axis, for the 15MA ITER baseline equilibrium. $s$ is square root of the normalized poloidal magnetic flux, labelling the plasma minor radius. 


\section{Computational model}

The MARS-F code, that we use in this work, assumes the single fluid plasma response model. This model has previously been shown to reproduce the experimental measurements (both perturbed field outside the plasma and the internal plasma displacement) in the DIII-D RMP experiments, as long as the plasma pressure remains well below the no-wall Troyon beta limit $[13,15]$. The fluid model, that we adopt to compute the RMP response, consists of single fluid and resistive MHD approximation [11]. The fluid equations for the plasma, with a given plasma equilibrium toroidal flow $V_{\mathbf{0}}=R \Omega \widehat{\boldsymbol{\phi}}$ ( $\mathrm{R}$ is the plasma major radius, $\widehat{\boldsymbol{\phi}}$ the unit vector along the geometric toroidal angle $\phi$ of the torus), are written for the perturbed quantities $\boldsymbol{\xi}, \boldsymbol{v}, \boldsymbol{b}, \boldsymbol{j}, \boldsymbol{p}$, representing the plasma displacement, perturbed velocity, magnetic field, current density and pressure, respectively

$$
\begin{gathered}
i\left(\Omega_{R M P}+n \Omega\right) \xi=v+(\xi \cdot \nabla \Omega) R \hat{\phi} \\
i \rho\left(\Omega_{R M P}+n \Omega\right) \boldsymbol{v}=-\nabla \boldsymbol{p}+\boldsymbol{j} \times \boldsymbol{B}+\boldsymbol{J} \times \boldsymbol{b}-\rho[2 \Omega \widehat{\boldsymbol{Z}} \times \boldsymbol{v}+(\boldsymbol{v} \cdot \nabla \Omega) R \widehat{\boldsymbol{\phi}}]-\nabla \cdot \boldsymbol{\Pi} \\
i\left(\Omega_{R M P}+n \Omega\right) \boldsymbol{b}=\nabla \times(\boldsymbol{v} \times \boldsymbol{B})+(\boldsymbol{b} \cdot \nabla \Omega) R \widehat{\boldsymbol{\phi}}-\nabla \times(\eta \boldsymbol{j}) \\
i\left(\Omega_{R M P}+n \Omega\right) \boldsymbol{p}=-\boldsymbol{v} \cdot \nabla \boldsymbol{P}-\Gamma \boldsymbol{P} \nabla \cdot \boldsymbol{v} \\
\boldsymbol{j}=\nabla \times \boldsymbol{b}
\end{gathered}
$$

where $\Omega_{R M P}$ is the rotation frequency of the RMP field, chosen to be $1 \mathrm{~Hz}$ in this study. The $1 \mathrm{~Hz}$ rotating field has been considered in the design of the ELM control system in ITER, for avoiding excessive (localized) heating flux load onto the divertor target [35]. $n$ is the toroidal harmonic number. We will consider $n=1-4$ in further investigations. $\hat{Z}$ is the unit vector in the vertical direction in the poloidal plane. The plasma resistivity is denoted by $\eta$. The Spitzer model is used for the plasma resistivity, which gives the on-axis Lundquist number of $8 \times 10^{9}$ for this ITER plasma. $\rho, \boldsymbol{B}, \boldsymbol{J}$ and $\boldsymbol{P}$ are the equilibrium plasma density, magnetic field, current density and pressure, respectively. $\Gamma=5 / 3$ is the ratio of specific heats.

The toroidal rotation frequency of the plasma is denoted as $\Omega$ in the above equations. In this work, we use the toroidal rotation as that predicted by the transport simulations and shown in Ref. [23] for the ITER plasma considered here. The plasma core rotation frequency is about $14 \mathrm{krad} / \mathrm{s}$, with the radial profile shown in Fig. 15(a) of Ref. [23]. It is understood that there is a large uncertainty associated with the prediction of the toroidal rotation in ITER. Fortunately, the plasma response has been found to be not very sensitive to the variation of the plasma flow speed, as long as the latter does not vary by one order of magnitude [36].

The last term in Eq.(2), $\nabla \cdot \boldsymbol{\Pi}=\rho \kappa_{\|}\left|k_{\|} \boldsymbol{v}_{\boldsymbol{t h \boldsymbol { i }}}\right|\left[\boldsymbol{v}+(\xi \cdot \nabla) \boldsymbol{V}_{\mathbf{0}}\right]_{\|}$, represents a viscous term describing the parallel sound wave damping physics. $\boldsymbol{\Pi}$ is a viscous stress tensor and $\kappa_{\|}$is the parallel sound wave damping coefficient. The value of $\kappa_{\|}$is set at 1.5 in this work, corresponding to a strong sound wave damping.

The ITER double vacuum vessel is also included and modelled as thin shells. In the vacuum region, the perturbed velocity $\boldsymbol{v}$ and pressure $\boldsymbol{p}$ vanish. The perturbed magnetic field satisfies 


$$
\nabla \times \boldsymbol{b}=0, \nabla \cdot \boldsymbol{b}=0
$$

The magnetic field due to the source current $\boldsymbol{j}_{\boldsymbol{R} M \boldsymbol{P}}$ is given as follows:

$$
\nabla \times \boldsymbol{b}=\boldsymbol{j}_{R M P}, \nabla \cdot \boldsymbol{j}_{R M P}=0
$$

where $\boldsymbol{j}_{\boldsymbol{R} M \boldsymbol{P}}$ represents the (surface) current density flowing the RMP coils, varying as $\exp (\operatorname{in} \phi)$ along the toroidal angle $\phi$. For the given RMP coil current source, the steady state solution of Eqs. (1-7) represents the plasma response in the linear model. It is clear that the response amplitude is proportional to that of the RMP currents.

\section{Computational results of plasma response versus coil geometry variation}

\subsection{Specification of figures of merit}

Evidently the plasma response can be measured by many quantities. In this work, we shall mainly consider two of them, which we shall further refer to as figures of merit. The first is the amplitude of the pitch resonant poloidal Fourier harmonic for the perturbed radial magnetic field near the plasma edge. More precisely, with a slight numerical smoothing of the plasma boundary shape near the X-point, and thus the presence of a finite safety factor $q_{a}$ at the plasma surface, we choose the resonant component computed at the outermost rational surface inside the plasma. This is not a unique but representative choice, since the final conclusion is sensitive neither to the smoothing/truncation of the plasma boundary, nor to the choice of the rational surfaces, as long as they are sufficiently close to the plasma surface [25]. The amplitude of the radial field component, in a straight field line coordinate system (chosen as a PEST-like coordinate system in this study, with the jacobian for the coordinates transformation being proportional to $R^{2}$ ), is defined as

$$
b_{r e s}^{1}=\left|\left(\frac{\boldsymbol{b} \cdot \nabla \psi}{\boldsymbol{B}_{\text {eq }} \cdot \nabla \phi}\right)_{m \boldsymbol{m}}\right| \frac{1}{R_{0}^{2}}
$$

where $\boldsymbol{b}$ is perturbed magnetic field, $\psi$ the equilibrium poloidal magnetic flux, $\boldsymbol{B}_{\boldsymbol{e q}}$ the equilibrium field, $R_{0}$ the major radius of the torus. For a given $n$ number ( $n=1-4$ in this work), the poloidal harmonic $m$ is chosen, such that $q=m / n$ is largest rational number below $q_{a}$. Further on, we shall compute and compare this figure of merit for both the vacuum RMP field alone and the total field perturbation including the resistive plasma response. However, for the purpose of the RMP coil geometry optimization, we shall mainly consider $b_{\text {res }}^{1}$ including the plasma response, since this has been shown to be a more relevant factor indicating the ELM mitigation in present experiments [37].

The second figure of merit is defined as magnitude of the normal displacement $\xi_{n}$ of the plasma surface near the X-point. The plasma surface displacement, representing 3D corrugation of the plasma boundary, is caused by the plasma response to the applied RMP field. Previous modelling results on existing devices $[16,25,31,32]$ have shown that the X-point displacement also 
serves as a good indicator for the ELM mitigation and/or the accompanied plasma density pump out effect. For the purpose of comparison, in this study, we shall also consider the plasma surface displacement near the outboard mid-plane. It is important to point out that, unlike $b_{\text {res }}^{1}$, the plasma surface displacement is a reasonably easily measurable quantity in experiments $[18,20]$.

In the following, we shall report the aforementioned figures of merit computed by MARS-F, for the ITER equilibrium, while systematically scanning the RMP coil geometry parameters as shown in Fig. 1. The computations are performed assuming the toroidal numbers $n=1,2,3,4$ for the coil configuration. We first illustrate the investigation for $n=3$, using upper and lower rows of coils.

\subsection{Plasma response to upper and lower rows of RMP coils with $n=3$}

As mentioned before, in the presence of two rows of coils, one more degree of freedom for the choice of the coil configuration is the toroidal phase difference of the coil currents between two rows. In other words, defining the lower row coil current of a wave form $I_{\text {lower }} \propto \cos \left(n \phi_{\text {coil }}\right)$, the upper row follows as $\cos \left(n \phi_{\text {coil }}+\Delta \Phi_{U L}\right)$. Here $\phi_{\text {coil }}$ is the toroidal angle for the location of the center of the coil [18]. This phase difference $\Delta \Phi_{U L}=\Phi_{U}-\Phi_{L}$, further referred to as the coil phasing, can be continuously varied if independent power supply is available for each coil, as is the case for the ITER RMP design. In this study, we limit ourselves with three representative choices for the coil phasing, namely $\Delta \Phi_{U L}=0^{\circ}$ (even parity), $\Delta \Phi_{U L}=180^{\circ}$ (odd parity) and $\Delta \Phi_{U L}=90^{\circ}$.
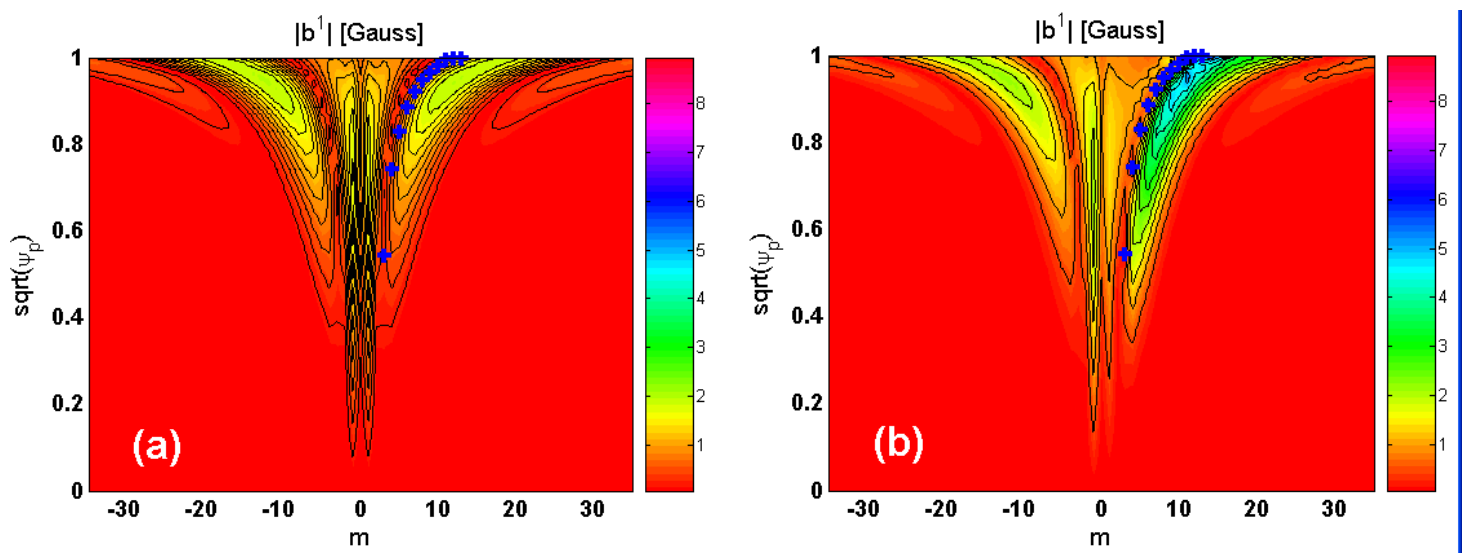

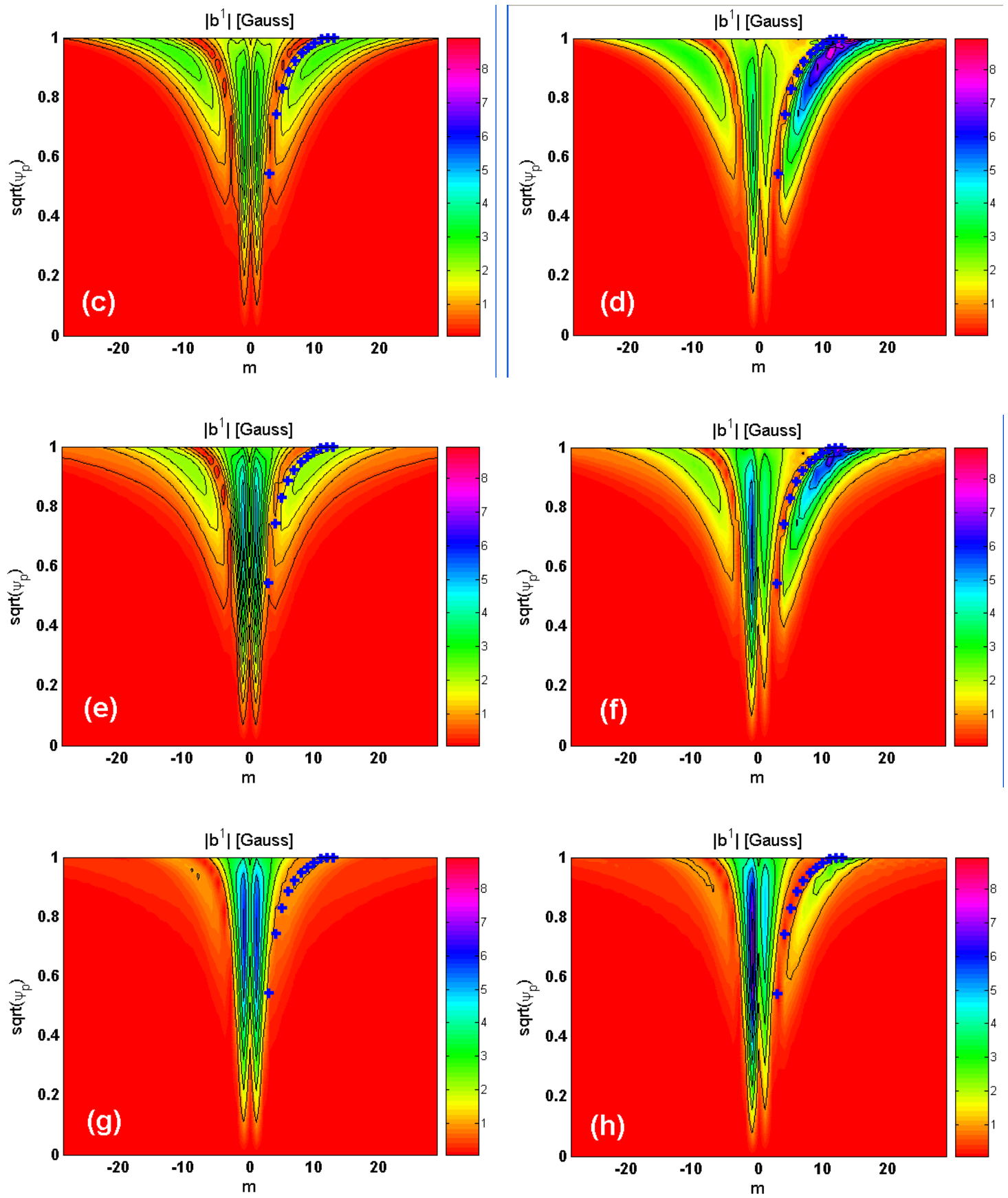

Figure 3. Comparison of the poloidal spectra in the full plasma region, between the vacuum radial field (left panel) and the total radial field including the plasma response (right panel), for the coils' width $(a, b) \Delta \theta=18^{\circ},(c, d) \Delta \theta=36^{\circ},(e, f) \Delta \theta=54^{\circ}$, and $(g, h) \Delta \theta=72^{\circ}$, respectively, using upper and lower rows of coils located at $\theta_{c}^{U}=39.0^{\circ}$ and $\theta_{c}^{L}=-41.1^{\circ}$, respectively, in the even parity (the toroidal phasing of the coil currents $\Delta \Phi_{U L}=0^{\circ}$ ) $n=3$ configuration with $45 \mathrm{kAt}$ current. The symbols ' + ' indicate the location of the $q=m / n$ rational surfaces.

Figure 3 shows a series of examples for the computed radial field spectrum, assuming the even parity, $n=3$ coil configuration for the upper and lower rows of coils. The poloidal locations of the coils are fixed at the ITER design value. The poloidal coverage of the coils, on the other hand, are gradually increased. For each coil width, compared are also the radial field spectra between the vacuum field (left panel) and the total field including the plasma response (right panel). The color 
coding shows the amplitude of the radial field, for both resonant and non-resonant components. Note the apparent symmetric distribution of the vacuum field spectra, which is broken by the plasma response, largely due to the amplification of the off-resonant part of the spectrum. This amplification, usually identified as the kink response [16,21,24,25], is rather strong for the low $n$ $(n=1,2)$ coil configuration, but is weak for higher $n$ 's $(n=4)$, for this ITER plasma. Another apparent effect is the reduction of the high-m spectra near the plasma edge, with increasing coil width. This is more clearly seen in the vacuum field spectra shown in the left panel.

The locations of all the rational surfaces, $q=m / n$, in this spectrogram are marked by the "+" symbols in Fig. 3. With the poloidal location of the upper and lower rows coils as designed in ITER, and assuming the even parity, the $n=3$ resonant spectra are always off the peak values in the spectrogram independent of the choice of the coil width, even for the vacuum field. The plasma response generally reduces the amplitude of the resonant harmonics in the core region, due to the plasma response induced screening effect [11]. However, the resonant harmonics near the plasma edge can either be reduced or amplified, compared to the corresponding vacuum field, by the plasma response, depending on the coil's geometry. For the case considered in Fig. 3, the resonant field components are amplified by the plasma response, largely due to the coupling effect between the resonant and the (amplified) non-resonant spectrum [25]. This amplification of the resonant components, near the plasma edge, will be more clearly shown by Fig. 4 .

Similar computations, to that shown in Fig. 3, are performed with more systematic scan of the coils poloidal location $\theta_{c}$ and poloidal coverage $\Delta \theta$. The results, in terms of the figures of merit as defined in sub-section 4.1, are reported in Figs. 4-6. In particular, Fig. 4 shows the amplitude of the last resonant radial field perturbation $b_{r e s}^{1}$ versus the variation of either the poloidal location of the coils (left panel), or the poloidal coverage of the coils (right panel). These parameters are symmetrically varied for the upper and lower rows of coils, i.e. $\theta_{c}^{U}=-\theta_{c}^{L}$, $\Delta \theta^{U}=\Delta \theta^{L}$. At fixed $\Delta \theta$, the plasma response (solid curves) can either amplify or reduce the last resonant harmonic, compared to that of the vacuum field (dashed curves), as shown by the left panel of Fig. 4. On the other hand, at fixed $\theta_{c}$, it appears that variation of the coil width does not lead to substantial alternation of between amplification and reduction of the resonant field amplitude. For instance, at even parity (Fig. 4(b)), the plasma response generally always amplifies the resonant harmonics, compared to the vacuum field approximation.

In Fig. 4, the corresponding ITER design values for $\theta_{c}^{U},-\theta_{c}^{L}, \Delta \theta^{U}$, and $\Delta \theta^{L}$, are indicated by vertical lines. The designed coil locations $\theta_{c}$ are generally not far from the optimal values that maximize the pitch resonant radial field component including the plasma response. On the other hand, choice of the coil width $\left(\sim 15^{\circ}\right)$ in the ITER design is sub-optimal. The best coil width, for maximizing $b_{\text {res }}^{1}$ including the plasma response, is $\Delta \theta \sim 40^{\circ}$. 

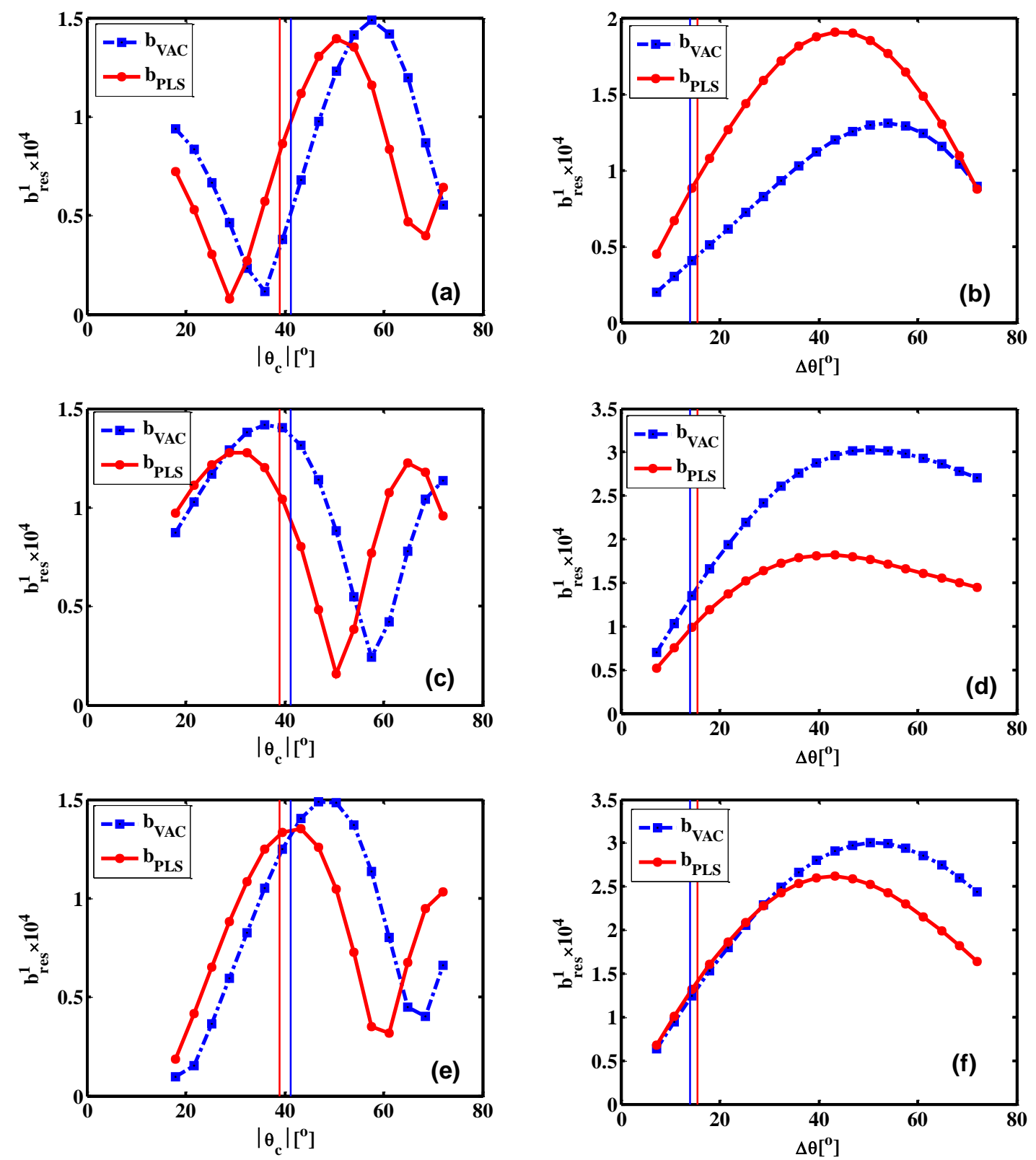

Figure 4. (Color online) Comparison of the amplitude of the last pitch resonant radial field component, in [Tesla], between the vacuum field (dashed) and the total field including the plasma response (solid), versus the coils' poloidal location $\left|\theta_{c}\right|$ (left panel, at fixed $\Delta \theta=15.5,14.1$ ) and the coils' width $\Delta \theta$ (right panel, at fixed $\theta_{c}=39.0,-41.1$, for the toroidal phasing of the coil currents at $(a, b) \Delta \Phi_{U L}=0^{\circ}$ (even parity), $(c, d) \Delta \Phi_{U L}=180^{\circ}$ (odd parity) and $(e, f) \Delta \Phi_{U L}=$ $90^{\circ}$, respectively, using upper and lower rows of coils in the $n=3$ configuration with $45 \mathrm{kAt}$ current. Vertical lines indicate the ITER design values for the coils $\left(\theta_{c}^{U}=39.0^{\circ},-\theta_{c}^{L}=41.1^{\circ}\right.$, $\Delta \theta^{U}=15.5^{\circ}$, and $\left.\Delta \theta^{L}=14.1^{\circ}\right)$.

Similar conclusion can be reached by using the second figure of merit, namely the plasma displacement (in particular the X-point displacement) shown in Fig. 5. In other words, the coils poloidal locations from the ITER design are close to maximizing the plasma displacement near the X-point, but the coils width appears to be sub-optimal. It should be noted that the choice of the poloidal extension of the RMP coils is often limited by engineering and other constraints. Figures 4-5 (right panels) offer quantitative measures on how far the design is from the optimum, if these 
constraints were absent.
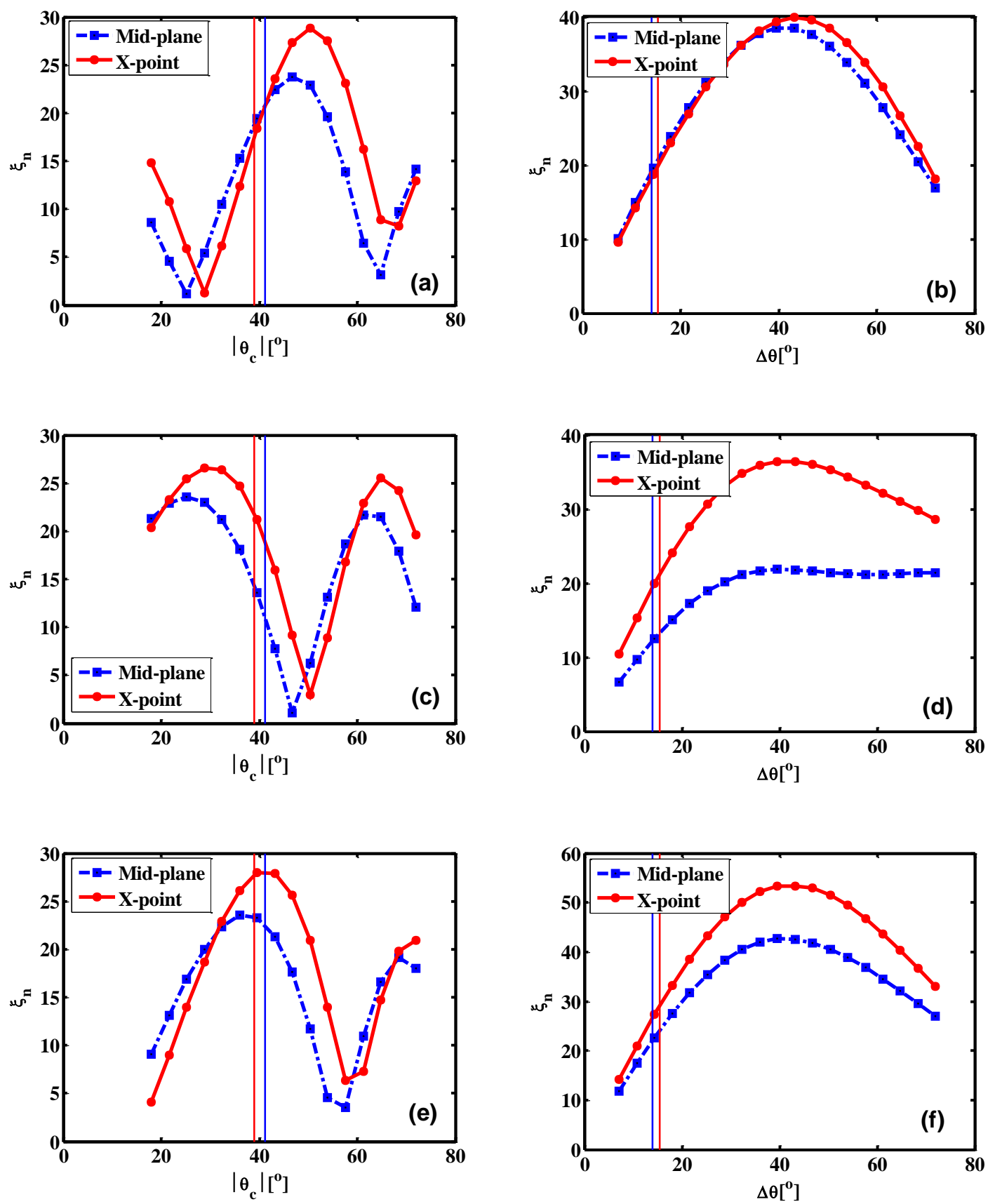

Figure 5. (Color online) Comparison of the amplitude of the plasma surface displacement, in [mm], at the low field side mid-plane (dashed) and near the X-point (solid), versus the coils' poloidal location $\left|\theta_{c}\right|$ (left panel, at fixed $\Delta \theta=15.5,14.1$ ) and the coils' width $\Delta \theta$ (right panel, at fixed $\theta_{c}=39.0,-41.1$, for the toroidal phasing of the coil currents at $(a, b) \Delta \Phi_{U L}=0^{\circ}$ (even parity), (c,d) $\Delta \Phi_{U L}=180^{\circ}$ (odd parity) and $(e, f) \Delta \Phi_{U L}=90^{\circ}$, respectively, using upper and lower rows of coils in the $n=3$ configuration with $45 \mathrm{kAt}$ current. Vertical lines indicate the ITER design values for the coils $\left(\theta_{c}^{U}=39.0^{\circ},-\theta_{c}^{L}=41.1^{\circ}, \Delta \theta^{U}=15.5^{\circ}\right.$, and $\left.\Delta \theta^{L}=14.1^{\circ}\right)$.

It is interesting to note that both the figures of merit - the amplitude of the last pitch resonant radial field component and the plasma X-point displacement - lead to very similar predictions for the optimal coil geometry. Indeed, both criteria seems to be reliable in interpreting the observed ELM mitigation by RMP coils in experiments [25,37]. Figure 6 selects and compare these two 
figures of merit in the same place, for three choices of the coil phasing. The strong similarity is evident.
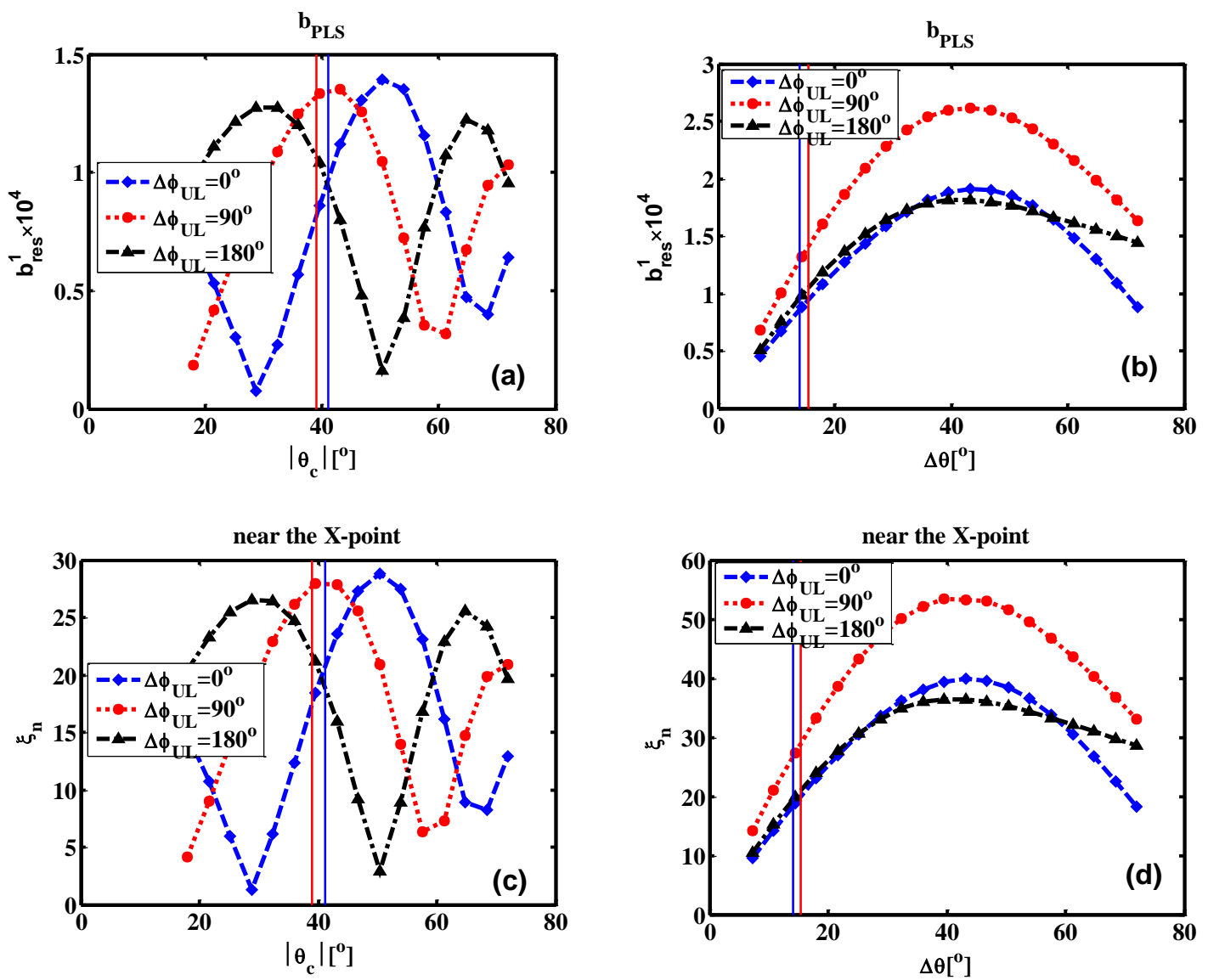

Figure 6. (Color online) Comparison of $(a, b)$ the amplitude, in [Tesla], of the last pitch resonant radial field component including the plasma response, and $(c, d)$ the plasma surface displacement, in [mm], near the X-point, versus the coils' poloidal location $\left|\theta_{c}\right|$ (left panel, at fixed $\Delta \theta=$ $15.5,14.1)$ and the coils' width $\Delta \theta$ (right panel, at fixed $\theta_{c}=39.0,-41.1$ ), for the toroidal phasing of the coil current $\Delta \Phi_{U L}=0^{\circ}$ (even parity), $\Delta \Phi_{U L}=90^{\circ}$, and $\Delta \Phi_{U L}=180^{\circ}$ (odd parity), using upper and lower rows of coils in the $n=3$ configuration with $45 \mathrm{kAt}$ current. Vertical lines indicate the ITER design values for the coils $\left(\theta_{c}^{U}=39.0^{\circ},-\theta_{c}^{L}=41.1^{\circ}\right.$, $\Delta \theta^{U}=15.5^{\circ}$, and $\Delta \theta^{L}=14.1^{\circ}$ ).

\subsection{Comparison of plasma response to upper and lower rows of coils with $n=1-4$}

With all 27 RMP coils independently powered in the ITER design, it is possible to achieve coil configurations with different dominant $n$-numbers. In this study, we consider $n$ up to 4 . We also neglect the sideband toroidal harmonics for a given $n$ configuration, although these sidebands (e.g. $n=5$ harmonics with the $n=4$ configuration) are not avoidable with limited number of coils ( 9 in ITER) along the toroidal angle. A comparative investigation of the plasma response with $n=1-4$, again with systematic scan of the coil geometry parameters, is carried out in this work, with results reported in Figs. 7-10. 

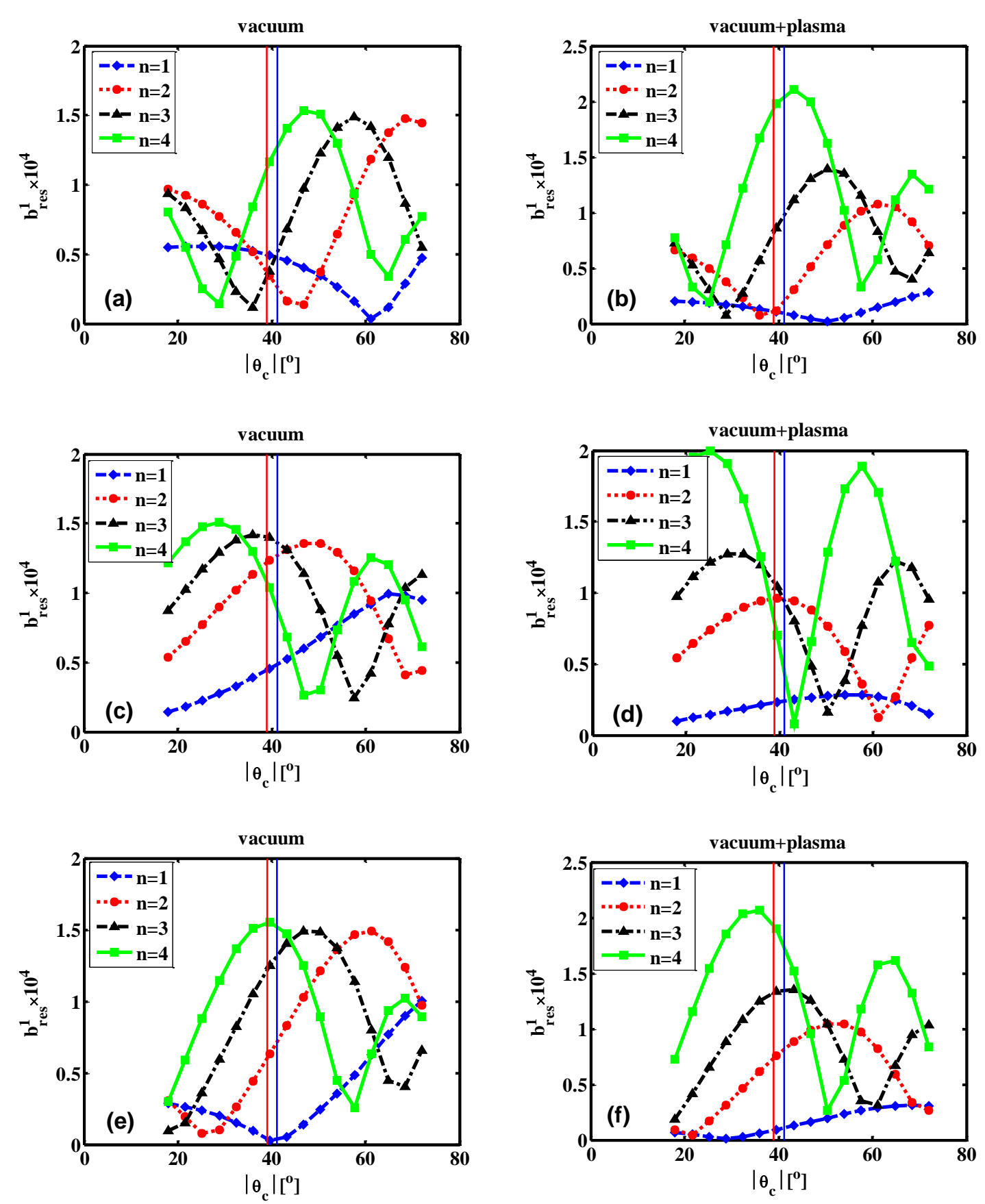

Figure 7. (Color online) Comparison of the amplitude, in [Tesla], of the last pitch resonant radial field component between the vacuum field (left panel) and the total field including the plasma response (right panel), versus the coils' poloidal location $\left|\theta_{c}\right|$ (at fixed $\Delta \theta=15.5,14.1$ ), for the toroidal phasing of the coil currents at $(a, b) \Delta \Phi_{U L}=0^{\circ}$ (even parity), $(c, d) \Delta \Phi_{U L}=180^{\circ}$ (odd parity) and (e,f) $\Delta \Phi_{U L}=90^{\circ}$, respectively, using upper and lower rows of coils in the $n=$ $1,2,3,4$ configurations with $45 \mathrm{kAt}$ current. Vertical lines indicate the ITER design values for the coils $\left(\theta_{c}^{U}=39.0^{\circ},-\theta_{c}^{L}=41.1^{\circ}\right)$.

Figure 7 shows the variation of $b_{r e s}^{1}$ versus $\theta_{c}$, for three choices of the coil phasing, again using the upper and lower rows of coils. Comparison is also made between the vacuum field (left panel) and total field including the plasma response (right panel). In each plot, the resonant field amplitude with different $n$ 's is compared. The coil currents are assumed to be the same (45kAt) for all $n$ 's. This leads to comparable peak amplitude (over $\theta_{c}$ ) of the vacuum resonant field component 
near the plasma edge, between different $n$ 's. The location of the peaks, however, vary with $n$. Essentially, with increasing the toroidal number for the RMP fields, the upper and lower rows of coils need to be shifted more towards the outboard mid-place, in order to produce the largest resonant field components.

Inclusion of the plasma response does not vary the aforementioned trend for the location of the field peaks. The amplitude of the peaks, on the other hand, is subject to more variations due to the plasma response. In particular, the $n=4$ response results in the largest peak values, for all three coil phasing considered here. The $n=1$ resonant field, on the other hand, is subject to the strongest screening. In terms of maximizing the plasma response based $b_{r e s}^{1}$, the present ITER coil design is not far from the optimum, almost for all cases but two - the $n=2$ even parity and the $n=4$ odd parity configurations. In the latter two cases, the resonant field amplitude, including the plasma response, is close to the minimum. This shows that, with the poloidal location of the coils as designed in ITER, and the given toroidal mode number $n$ for the field perturbation, optimization of the coil phasing between the upper and lower rows may be important, in order to produce the best effects on ELMs.

Figure 8 shows the computed plasma surface displacement near the outboard mid-plane (left panel) and the X-point (right panel), for the same scan of the coils geometry and phasing, as in Fig. 7. Whilst no consistent trend is shown by the mid-plane displacement, the systematic shift of the displacement peak near the X-point, with increasing $n$, is evident. In fact, the optimal coils location, predicted by maximizing the X-point displacement, matches well with that predicted by maximizing the plasma response based $b_{r e s}^{1}$ shown in Fig. 7, again showing the consistency between these two figures of merit.
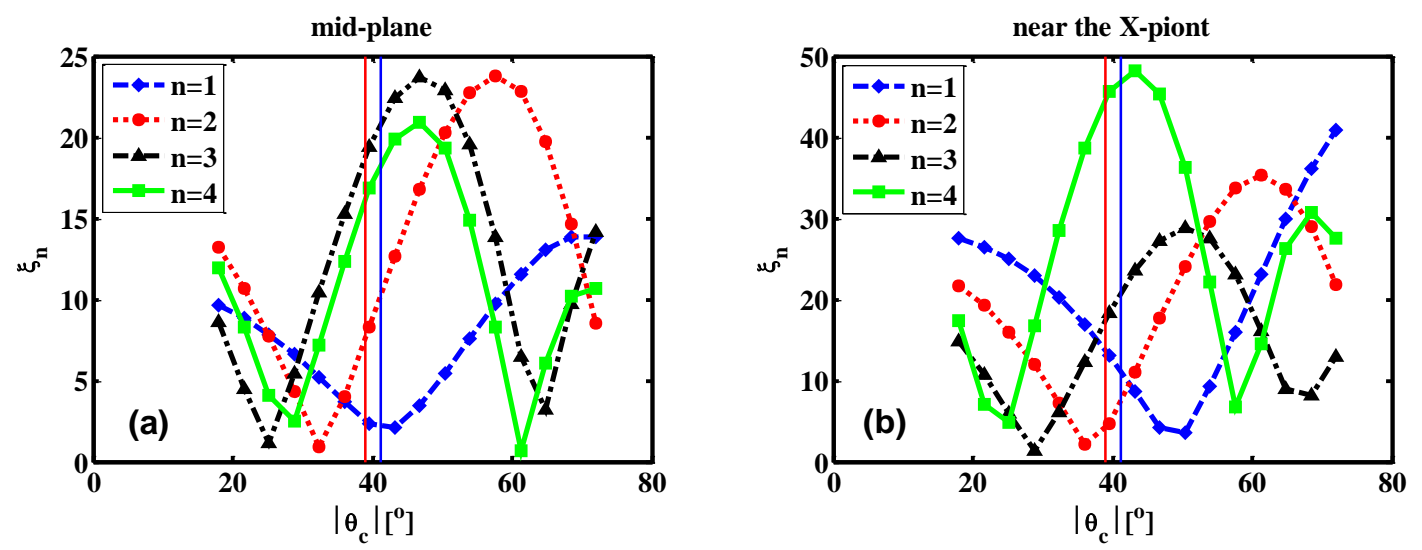

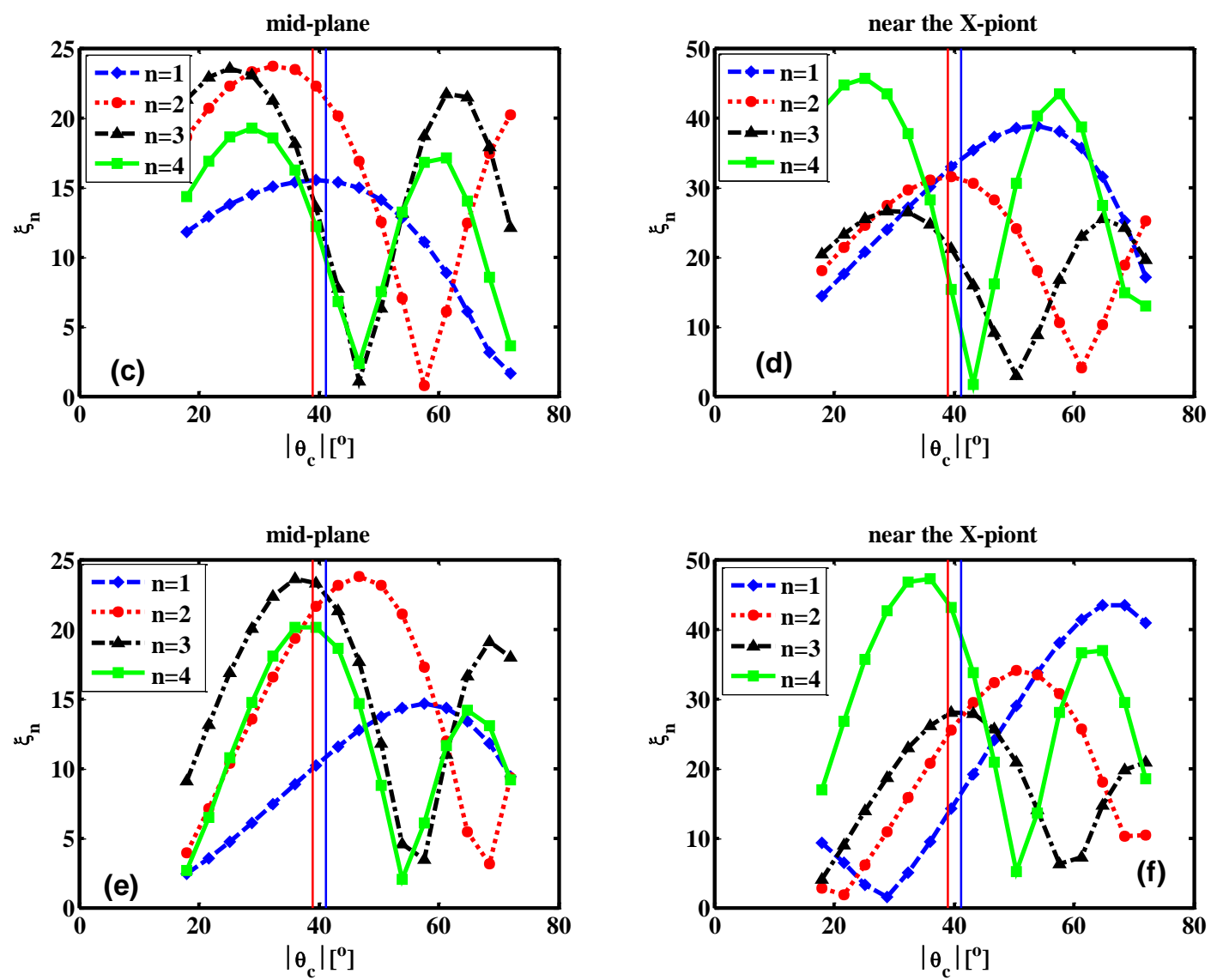

Figure 8. (Color online) Comparison of the amplitude of the plasma surface displacement, in [mm], at the outboard mid-plane (left panel) and near the X-point (right panel), versus the coils' poloidal location $\left|\theta_{c}\right|$ (at fixed $\Delta \theta=15.5,14.1$ ), for the toroidal phasing of the coil currents at $(a, b) \Delta \Phi_{U L}=0^{\circ}$ (even parity), $(c, d) \Delta \Phi_{U L}=180^{\circ}$ (odd parity) and $(e, f) \Delta \Phi_{U L}=90^{\circ}$, respectively, using upper and lower rows of coils in the $n=1,2,3,4$ configurations with $45 \mathrm{kAt}$ current. Vertical lines indicate the ITER design values for the coils $\left(\theta_{c}^{U}=39.0^{\circ},-\theta_{c}^{L}=41.1^{\circ}\right)$.

Figures 9-10 compare the figures of merit for $n=1-4$ coil configurations, while varying the poloidal coverage $\Delta \theta$ of each of the upper and lower rows of coils. The poloidal location $\theta_{c}$ of the coils are fixed at the ITER design values. With few exceptions (in particular for the $n=1$ case), the optimal coil width, maximizing these figures of merit, appears to be around $40^{\circ}$ (between $30^{\circ}-50^{\circ}$ ), for all three representative choices of the coil phasing. The coil width from the ITER design is again sub-optimal.
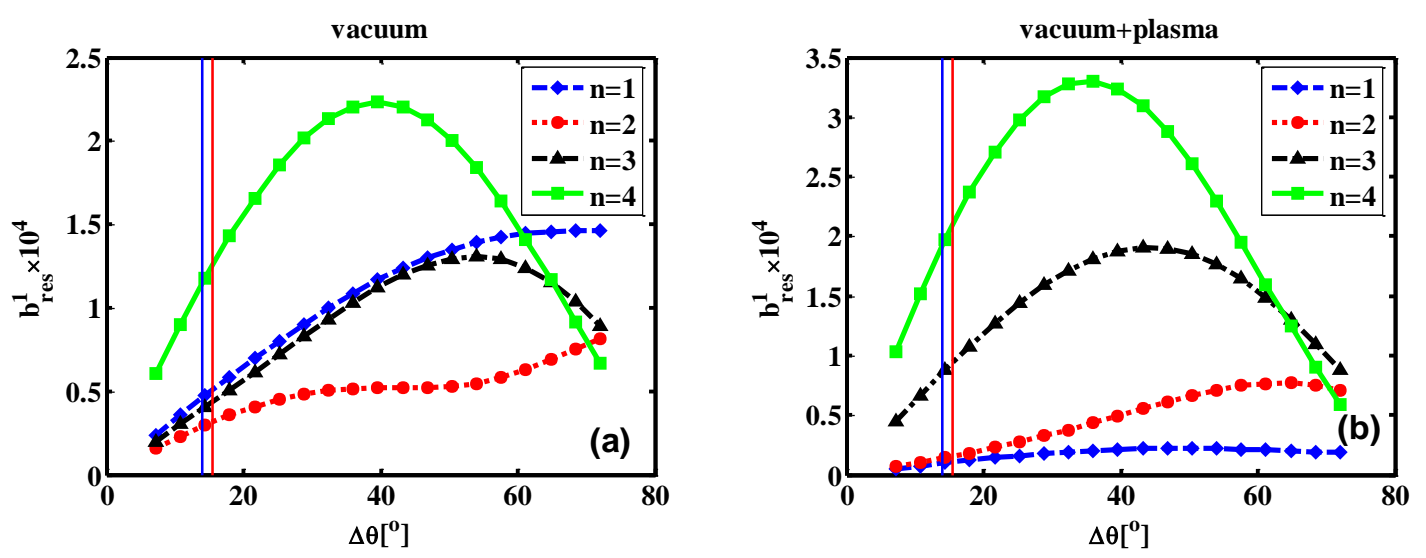

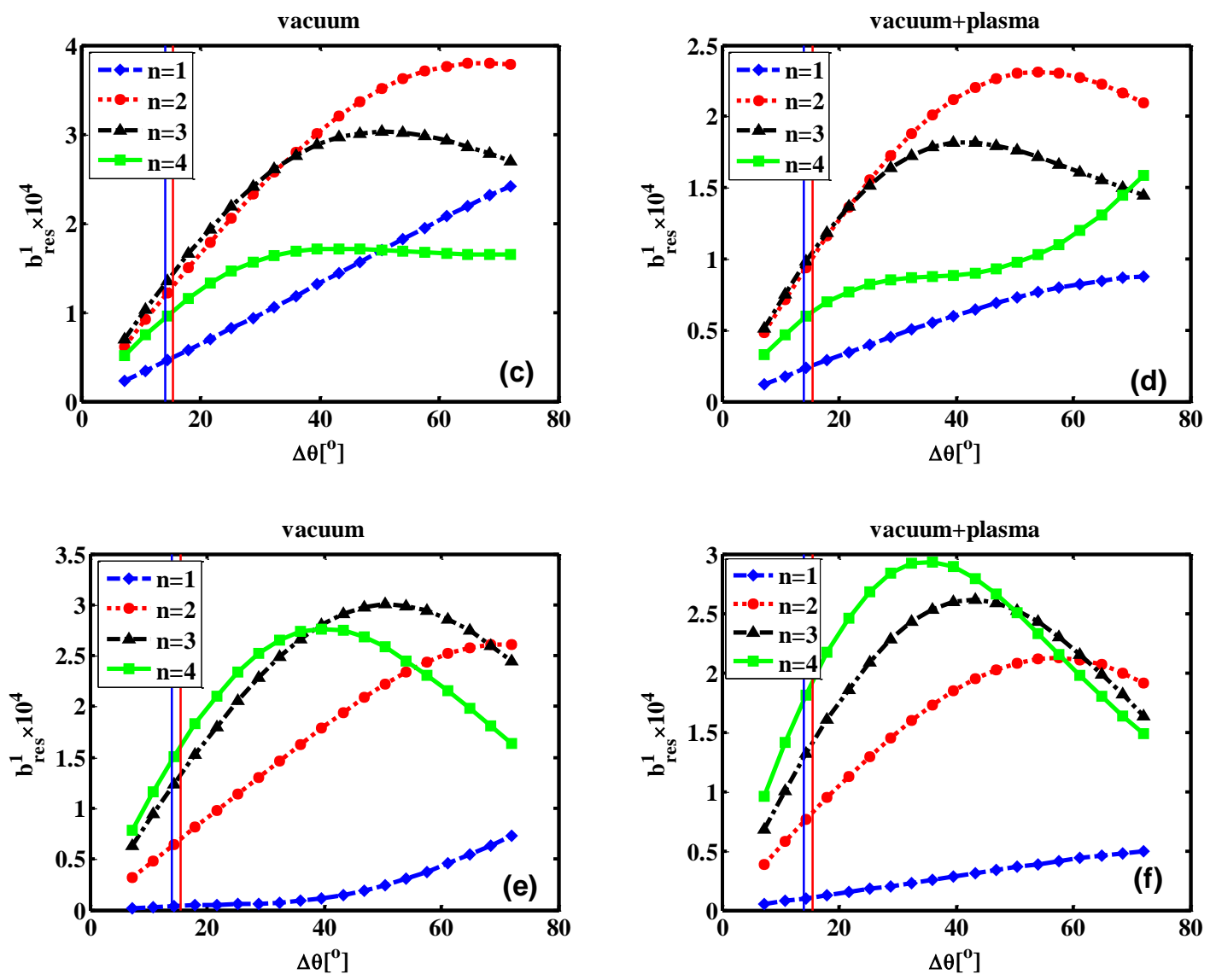

Figure 9. (Color online) Comparison of the amplitude, in [Tesla], of the last pitch resonant radial field component between the vacuum field (left panel) and the total field including the plasma response (right panel), versus the coils' poloidal width $\Delta \theta$ (at fixed $\theta_{c}=39.0,-41.1$ ), for the toroidal phasing of the coil currents at $(a, b) \Delta \Phi_{U L}=0^{\circ}$ (even parity), $(c, d) \Delta \Phi_{U L}=180^{\circ}$ (odd parity) and (e,f) $\Delta \Phi_{U L}=90^{\circ}$, respectively, using upper and lower rows of coils in the $n=$ $1,2,3,4$ configurations with 45kAt current. Vertical lines indicate the ITER design values for the coils $\left(\Delta \theta^{U}=15.5^{\circ}, \Delta \theta^{L}=14.1^{\circ}\right)$.
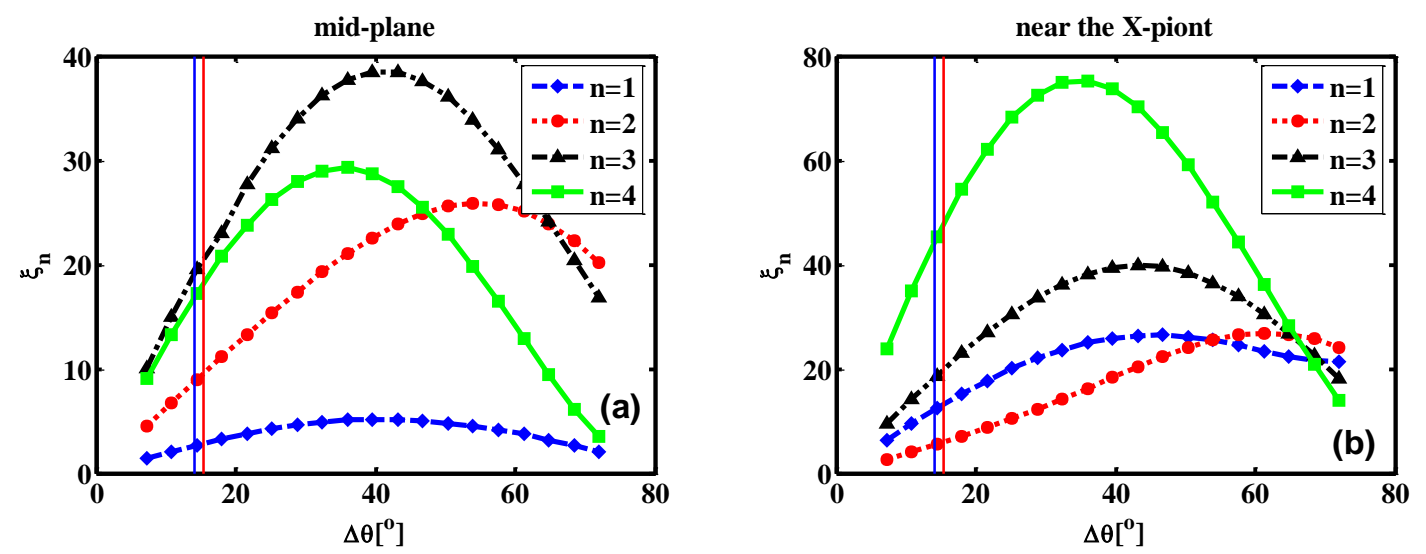

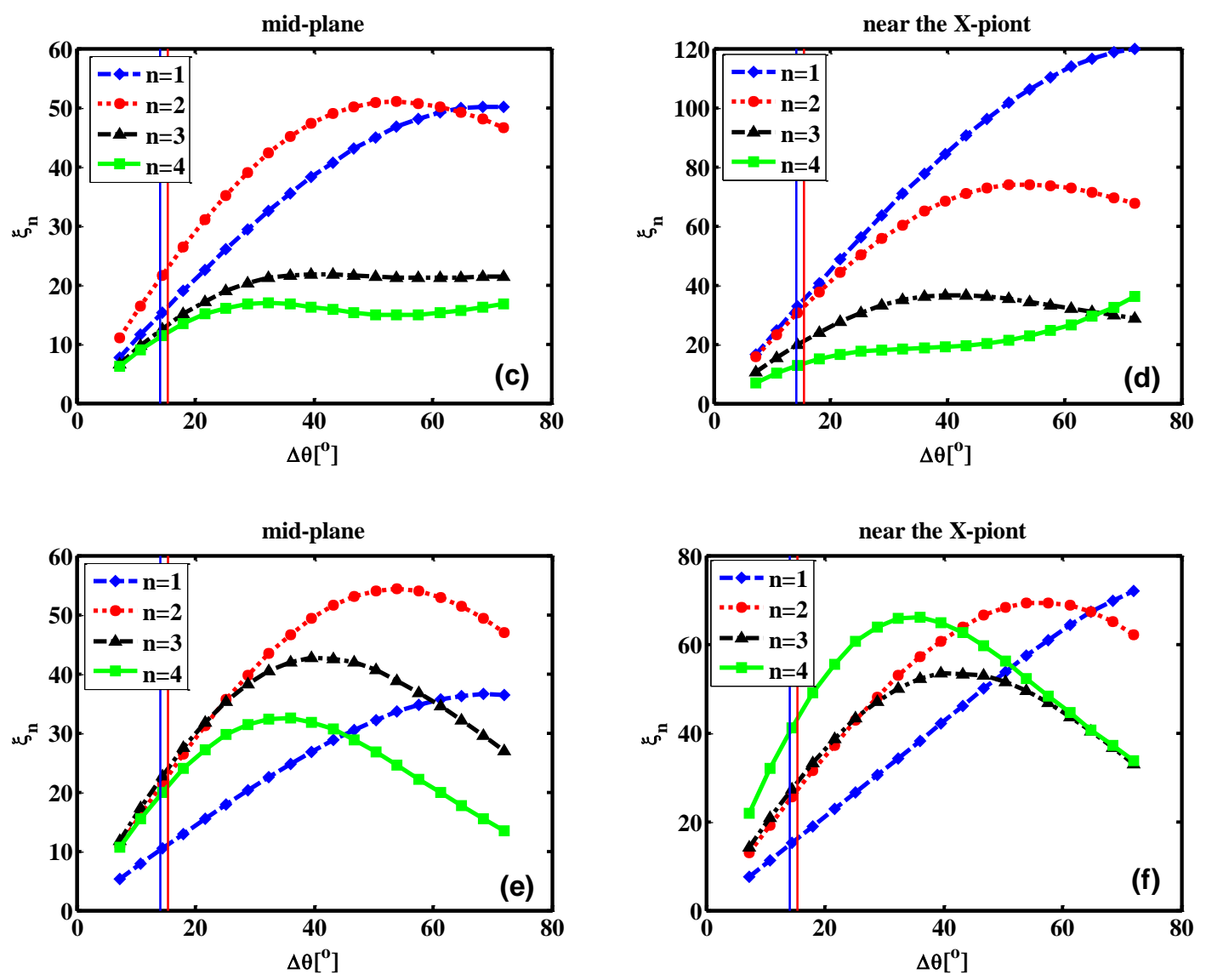

Figure 10. (Color online) Comparison of the amplitude of the plasma surface displacement, in [mm], at the outboard mid-plane (left panel) and near the X-point (right panel), versus the coils' poloidal width $\Delta \theta$ (at fixed $\theta_{c}=39.0,-41.1$ ), for the toroidal phasing of the coil currents at (a,b) $\Delta \Phi_{U L}=0^{\circ}$ (even parity), $(c, d) \Delta \Phi_{U L}=180^{\circ}$ (odd parity) and $(e, f) \Delta \Phi_{U L}=90^{\circ}$, respectively, using upper and lower rows of coils in the $n=1,2,3,4$ configurations with 45kAt current. Vertical lines indicate the ITER design values for the coils $\left(\Delta \theta^{U}=15.5^{\circ}, \Delta \theta^{L}=14.1^{o}\right)$.

\subsection{Plasma response to middle row of coils with $n=1-4$}

We report the computational results on the plasma response to the middle row of the RMP coil currents here. These coils are centered at the outboard mid-plane of the torus. The geometric factors, that we vary, are the radial location $r_{c}$ of the coils, as well as the poloidal width $\Delta \theta$ of the coils. By moving this row of coils radially, we assume that these coils can be placed inside or outside the ITER vacuum vessels (even in between the double wall). This is motivated by the fact $\underline{\text { that, in future devices, due to spatial constraint (lack of sufficient space between the blankets and }}$ the inner vacuum vessel) and/or engineering constraint (too strong irradiation damage to the coils due to large neutron flux), we may have to place the coils farther away from the plasma. Since we are assuming a nearly static RMP current (rotating at $1 \mathrm{~Hz}$ ), the induced eddy in the walls is very weak, practically not affecting the plasma response. Therefore, the major consequence of shifting the coils inside or outside the vacuum vessels is the variation of (i) the strength of the RMP field in the plasma region, and (ii) the poloidal spectrum of the applied field.

Figure 11 summarizes the results with systematic scan of all three factors $-r_{c}, \Delta \theta$, and $n$. The figures of merit considered here are $b_{\text {res }}^{1}$ for either the vacuum field (left panel) or the total 
field including the plasma response (right panel). Several interesting observations can be made. (i) the last pitch resonant field component always decays with the coils distance from the plasma, as expected. The decay rates are different for different $n$ 's - faster decay occurs for higher $n$. This also follows general expectations. It is more interesting to note that for each given $n$, the $b_{\text {res }}^{1}$ field sharply decays when moving the coils from inside the vacuum vessels to the outside of the vessels. Further increase of the coil radius (outside the vessels) does not substantially change the field strength anymore. This suggests that, even without considering the wall eddy current screening (e.g. for ac RMP fields at relatively high frequency), the proximity effect of the middle row RMP coils to the plasma surface is important in ITER. (ii) Comparing the plasma response field and the vacuum field, we find that screening of the applied field generally occurs for the low- $n$ (n=1-3) coil configurations, with the strongest screening occurs at $n=1$. However, slight amplification of the pitch resonant component of the applied field is observed for the $n=4$ coil configuration, due to the resistive plasma response. This is again due to the coupling of the resonant components to the non-resonant portion of the spectrum, which is amplified by the plasma due to the stable kink-peeling response, similar to the $n=3$ case with the upper and lower rows of coils in even parity (Figs. 3 and 4(b)). (iii) The final observation from Fig. 11 is the non-monotonic dependence of $b_{\text {res }}^{1}$ versus $\Delta \theta$ for higher $n(n=3,4)$ configurations. Similar observation has also been reported in the previous subsection, when the coil width is varied for the upper and lower rows of coils. For the low- $n(n=1,2)$ configurations, however, $b_{\text {res }}^{1}$ increases monotonically with the coil width.
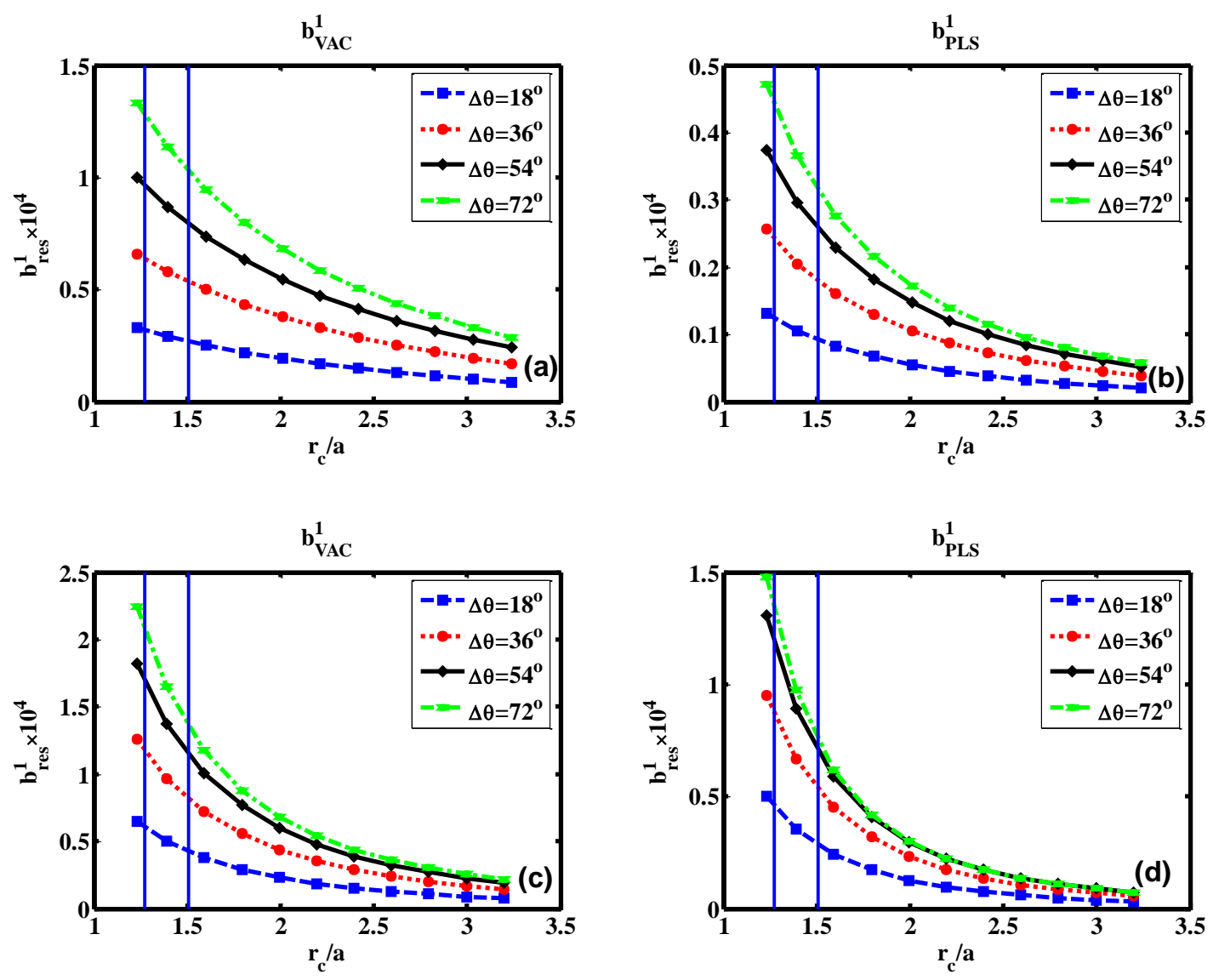

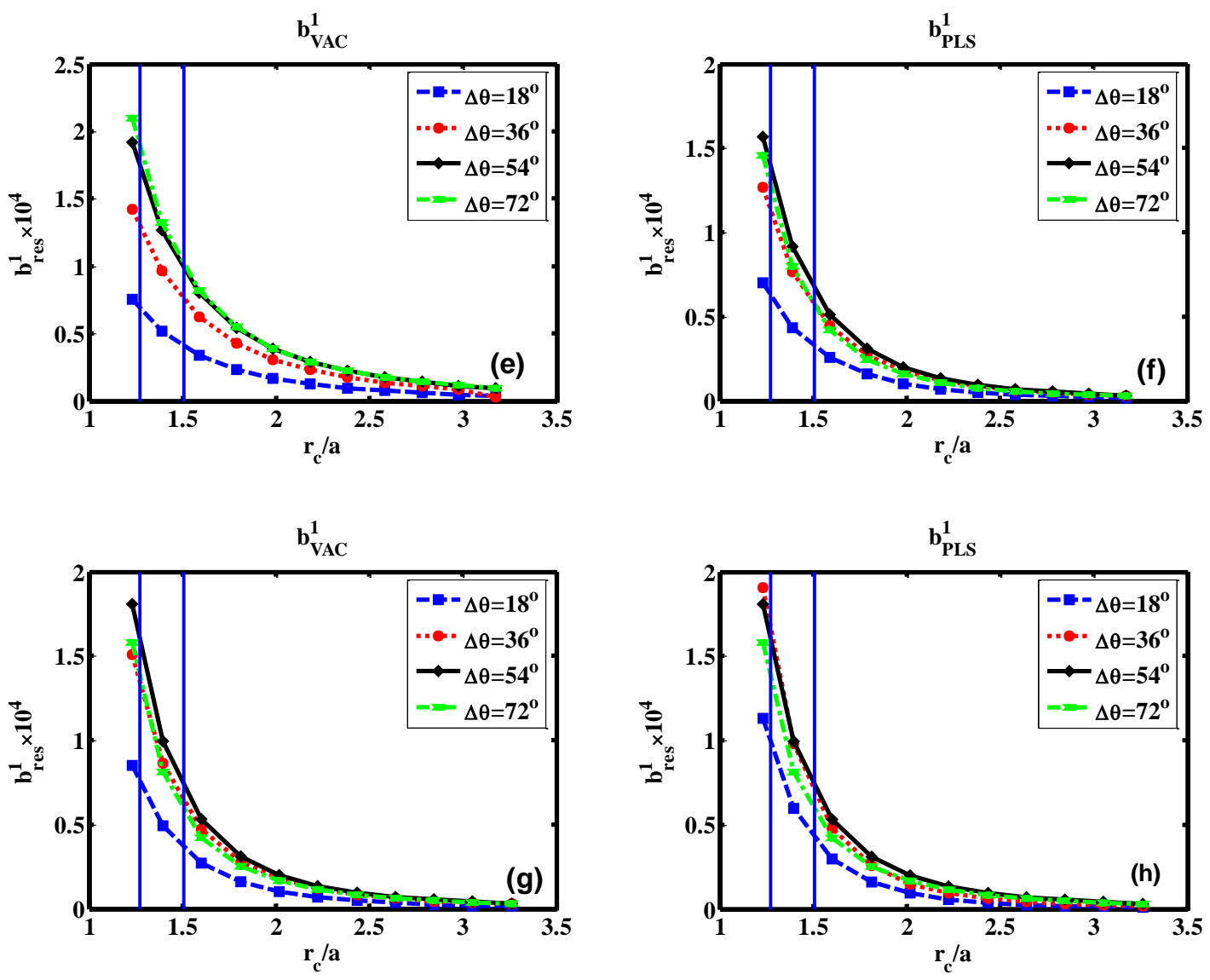

Figure 11. (Color online) Comparison of the amplitude, in [Tesla], of the last pitch resonant radial field component between the vacuum field (left panel) and the total field (total panel) including the plasma response, versus the radial location of the middle row coils, assuming $45 \mathrm{kAt}$ coil current in $(a, b) n=1,(c, d) n=2,(e, f) n=3$, and $(g, h) n=4$ configurations, respectively, and with different choices of the coils' poloidal width $\Delta \theta=18^{\circ}, \Delta \theta=36^{\circ}$, $\Delta \theta=54^{\circ}$, and $\Delta \theta=72^{\circ}$. The vertical lines indicate the radial locations of the ITER double wall.

All of the aforementioned observations are also generally confirmed by the computed plasma displacement near the X-point, as shown in Fig. 12. In addition, it is interesting to note the non-monotonic dependence of the displacement amplitude versus the toroidal mode number $n$, assuming the same coil current amplitude (45kAt) for each $n$. MARS-F computations predict that the largest $\mathrm{X}$-point displacement is caused by the middle row coils in the $n=1$ configuration. A large X-point displacement may be a good indicator of the RMP fields on the ELM mitigation [22]. But on the other hand, two large 3D plasma surface distortion may cause a potential problem of plasma locally touching the first wall in ITER. We note that nearly $7 \mathrm{~cm}$ plasma X-point displacement is computed for the $n=1$ configuration with the coil width of $\Delta \theta=72^{\circ}$, assuming the middle row coils are located just inside the vacuum vessels. The present ITER design for the middle row coils, with $\Delta \theta \sim 54^{\circ}$, can result in the X-point displacement of about $5 \mathrm{~cm}$. We also note that, for the $n=3$ and 4 configurations, the size of the middle row coils, as designed for ITER, is close to the optimum in maximizing both the resonant radial field (Fig. 11(f,h)) and the X-point displacement (Fig. 12(c-d)). 

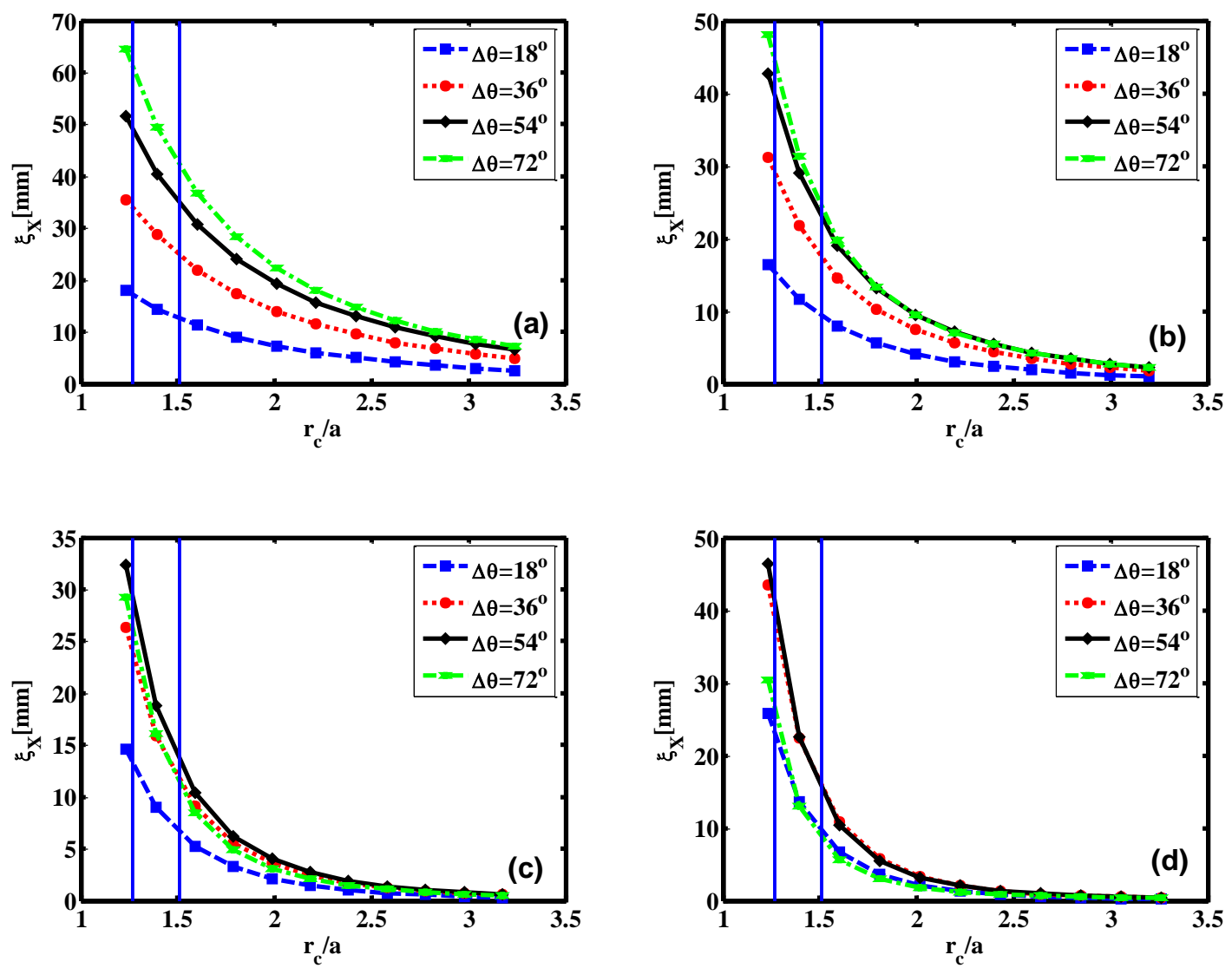

Figure 12. (Color online) Comparison of the amplitude of the plasma surface displacement near the $\mathrm{X}$-point versus the radial location of the middle row coils, assuming 45kAt coil current in (a) $n=1,(b) n=2$, (c) $n=3$ and (d) $n=4$ configurations, respectively, and with different choices of the coils' poloidal width $\Delta \theta=18^{\circ}, \Delta \theta=36^{\circ}, \Delta \theta=54^{\circ}$, and $\Delta \theta=72^{\circ}$. The vertical lines indicate the radial locations of the ITER double wall.

The 3D corrugation, due to the plasma response to the applied RMP fields, of course also occurs in other poloidal locations along the plasma boundary. Figure 13 shows the ratio of the $\mathrm{X}$-point to the outboard mid-plane displacements. It is interesting to note that, at low- $n(n=1,2)$ coil configurations, the mid-plane plasma displacement decays faster than the X-point displacement, while moving the middle row of coils away from the plasma. The opposite trend occurs at high- $n(n=4)$. The $n=3$ case represents an intermediate situation, where an optimal coil distance exists (at a fixed coil width), which maximizes the displacement ratio between the $\mathrm{X}$-point and the outboard mid-plane. We mention that this ratio has been shown also to serve as a good indicator of the observed density pump out in the RMP experiments in MAST [16]. 

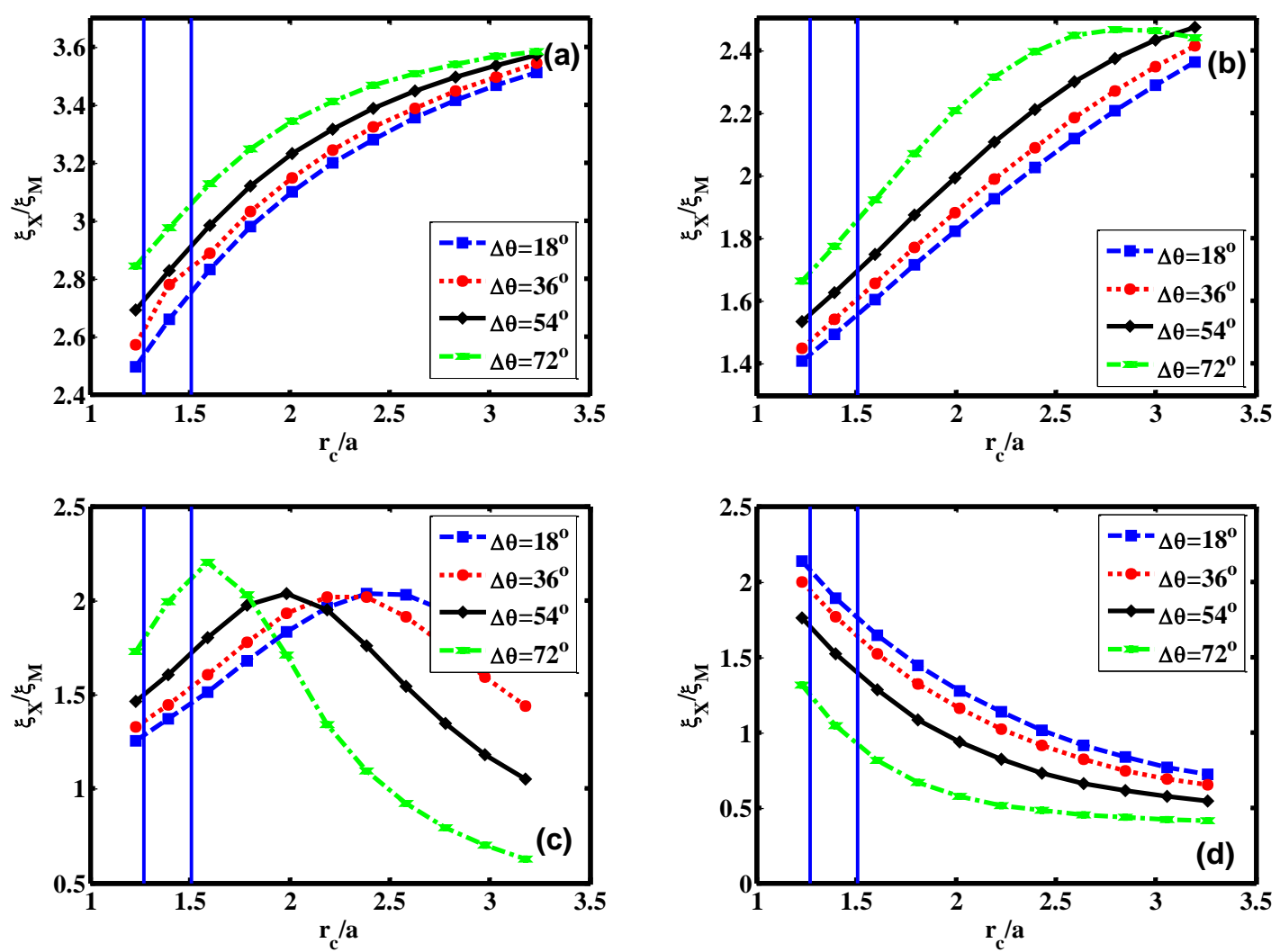

Figure 13. (Color online) Comparison of the ratio of the X-point to outboard mid-plane displacement of the plasma surface versus the radial location of the middle row coils, assuming 45kAt coil current in (a) $n=1,(b) n=2,(c) n=3$ and (d) $n=4$ configurations, respectively, and with different choices of the coils' poloidal width $\Delta \theta=18^{\circ}, \Delta \theta=36^{\circ}, \Delta \theta=54^{\circ}$, and $\Delta \theta=72^{\circ}$. The vertical lines indicate the radial locations of the ITER double wall.

\subsection{Plasma response to three rows of coils with $n=1-4$}

Below we consider several representative cases of the coil geometry optimization, when all three rows of coils are included. Due to a large number of degrees of freedom in the choice of optimization parameters, we restrict ourselves with certain assumptions. Specifically, (i) we fix the poloidal location as well as the width of the middle row coils as that designed for ITER; (ii) in terms of the coil phasing, we choose two limiting cases, one with zero phasing between the upper/lower rows and the middle row, and the other with $180^{\circ}$ phasing. This also implies that the upper and the lower rows are always in phase, which may not necessarily be the best choice for the ELM control. Our primary goal here is to study the coil geometry optimization. We leave more detailed coil current phase optimization to the future work.

For the above chosen coil phasing, we then vary either the poloidal location of the upper and lower rows of the coils (Fig. 14), at fixed coils width as from the ITER design, or vary the poloidal width of the upper and lower rows (Fig. 15), at fixed coils location as in the ITER design. As for the figure of merit, we choose the plasma surface displacement near the X-point.

For the $n=1$ or 2 configurations, the coils poloidal location from the ITER design is not optimal, with either $0^{\circ}$ or $180^{\circ}$ coil phasing (Fig. 14(a-b)). On the other hand, with the $n=3$ or 4 configurations, which are the preferable choices for the ELM control in ITER, the ITER coil design with $180^{\circ}$ phasing is close to optimal (Fig. 14(c-d)). The $0^{\circ}$ case represents the worst coil 
phasing for the ITER design, in terms of maximizing the plasma X-point displacement.

The poloidal width of the upper and lower row coils is sub-optimal for both choices of the coil phasing, for all $n=1-4$ (Fig. 15). In fact, it turns out the off-middle coils, of the similar size as the middle row coils, would be better, in particular for the $n=3$ and 4 configurations.

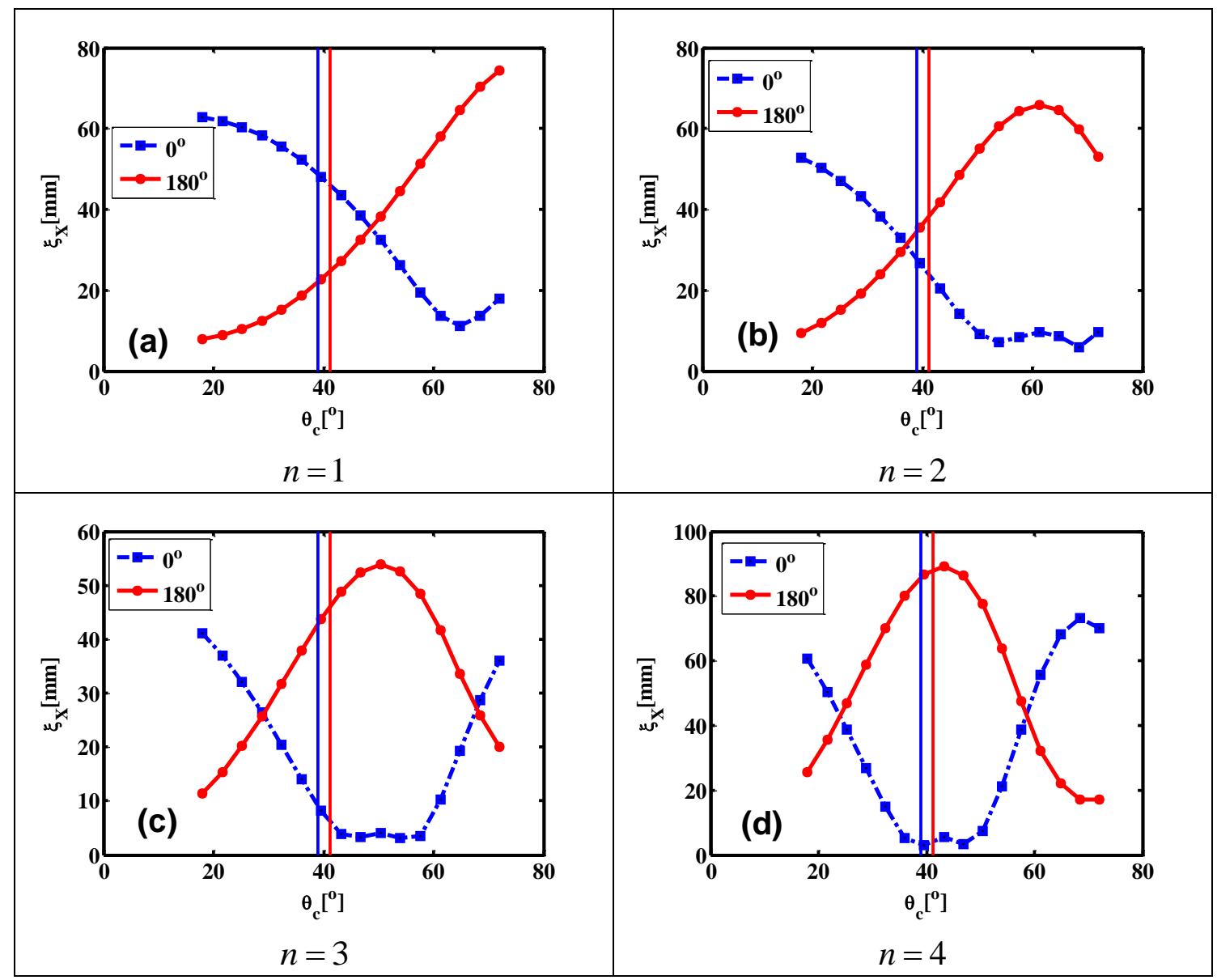

Figure 14. (Color online) Comparison of the amplitude of the plasma surface displacement near the X-point versus the coils' poloidal location $\left|\theta_{c}\right|$ (at fixed $\Delta \theta$ as from the ITER design for all 3 rows of coils), for the coil configuration of $n=1,2,3,4$ at $45 \mathrm{kAt}$, respectively. In each subplot, compared are also two cases with the relative toroidal phase difference of $0^{\circ}$ and $180^{\circ}$ between the upper/lower rows and the middle row coil currents. Vertical lines indicate the ITER design values for the upper and lower row coils $\left(\theta_{c}^{U}=39.0^{\circ},-\theta_{c}^{L}=41.1^{\circ}\right)$.

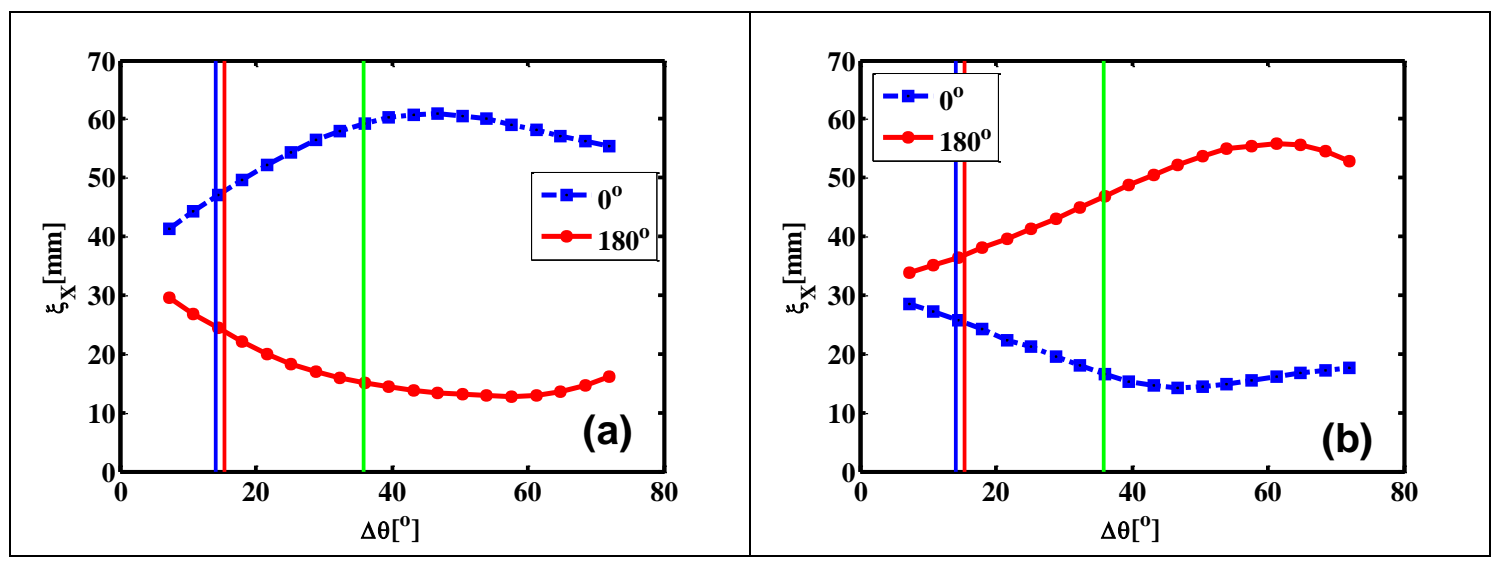




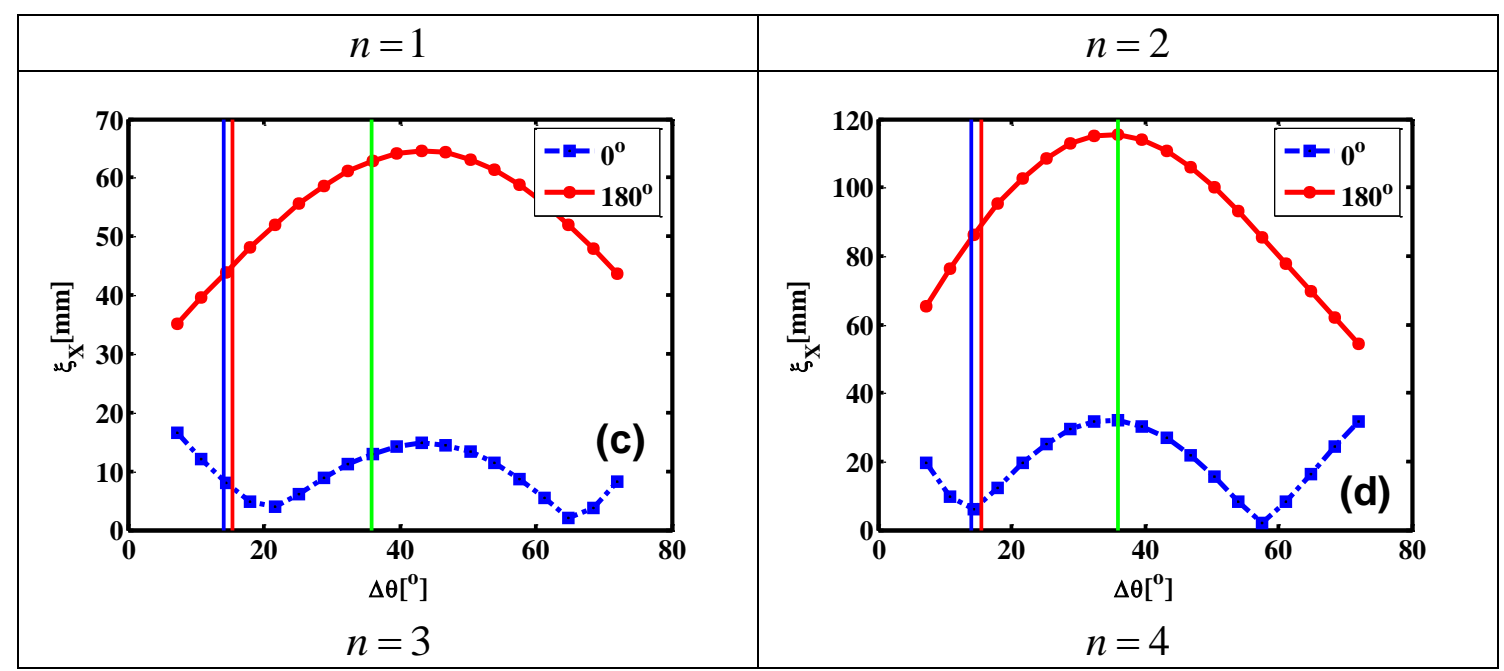

Figure 15. (Color online) Comparison of the amplitude of the plasma surface displacement near the X-point versus the coils' poloidal width $\Delta \theta$ for the upper and lower rows (at fixed $\theta_{c}$ as from the ITER design for all 3 rows of coils as well as fixed $\Delta \theta$ for the middle row coils), for the coil configuration of $n=1,2,3,4$ at $45 \mathrm{kAt}$, respectively. In each subplot, compared are also two cases with the relative toroidal phase difference of $0^{\circ}$ and $180^{\circ}$ between the upper/lower rows and the middle row coil currents. Vertical lines indicate the ITER design values for the poloidal width of three rows of coils, with the middle row being significantly larger than the upper and lower rows.

\section{Summary}

We have carried out a systematic numerical investigation of the effect of the RMP coil geometry on the plasma response, for an ITER 15MA baseline plasma. Full toroidal computations are performed using the MARS-F code, assuming $n=1,2,3,4$ coil configurations separately. Three ways of powering the RMP coils are exploited. The first way includes both the upper and lower rows of coils, with various toroidal phasing of the coil currents between the two, whilst the second considers only the middle row coils. In the third, we include all three rows of coils, but varying the poloidal location or width of the upper and lower rows - the coil size for the middle row is fixed at the ITER design value. With the upper and lower rows of coils, we vary both the location and coverage of each row along the (geometric) poloidal angle, while fixing the radial location of the coils just inside the inner vacuum vessel (beyond the blanket modules) of ITER as in the design. With the middle row, we vary the poloidal coverage, as well as the radial location of the coils. In all computations, the coil current, for each given toroidal mode number $n$, is assumed to be 45kAt. The results (i.e. the plasma response amplitude) scales linearly with the coil current.

We measure the plasma response by several figures of merit, namely the amplitude of the pitch resonant radial magnetic field component near the plasma edge, the MARS-F computed plasma surface displacement near the X-point and at the outboard mid-plane. In particular, the pitch resonant radial field amplitude near the plasma edge (including the plasma response), and the plasma displacement near the X-point, are chosen as the coil geometry optimization criteria, since previous research on existing tokamaks (MAST [16], ASDEX Upgrade [25], DIII-D [31], EAST [32]) has shown good correlation between maximizing these quantities on one side, and the 
observed ELM mitigation effects in experiments, on the other side. We find that both of these plasma response based criteria yield the same prediction for the optimal choices of the RMP coil geometry, for all cases considered in this work. This prediction differs from that based on the vacuum approximation. The highlights of the key findings, listed below, thus refer to the plasma response based criteria.

With both the upper and lower rows of coils powered, we considered three representative choices for the coil current phasing, i.e. $\Delta \Phi_{U L}=\Phi_{U}-\Phi_{U}=0^{\circ}$ (even parity), $180^{\circ}$ (odd parity) or $90^{\circ}$. With the $n=3$ coil configuration, we find that the poloidal location of the coils in the present ITER design is close to the optimum for all coil phasing. However, the poloidal coverage of the coils is sub-optimal. With the coil phasing of $\Delta \Phi_{U L}=90^{\circ}$, the ITER designed coils' poloidal location is close to optimum for $n=2,3,4$. On the other hand, this coils' location is far from optimum for the $n=2$ configuration at even parity, as well as for the $n=4$ configuration at odd parity. For all choices of the coil phasing and $n=1-4$, we find that the coils' poloidal width from the ITER design is sub-optimal but probably acceptable. The optimal choice of the coils' width, for both upper and lower rows of coils, is $\Delta \theta \sim 40^{\circ}$ as measured in geometric angle. Interestingly, adding the middle row coils as from the ITER design (i.e. considering all 3 rows of coils) does not qualitatively change the above conclusions.

With the middle row of RMP coils alone, the MARS-F computations show a sharp decrease of the plasma response amplitude (both in terms of the pitch resonant radial field component and the X-point displacement), as the coils move radially outwards, from the presently designed location (just inside the inner vacuum vessel) to a position of $r_{c} \sim 1.5 a$ (outside the outer vacuum vessel). This means that, with the same coil current, the efficiency of the ELM control may be significantly reduced by moving the middle row coils from the presently designed radial location to a position outside the ITER vacuum vessel. The rate of the sharp decay of the plasma response depends on the poloidal coverage $\Delta \theta$ of the coils. However, this influence becomes less pronounced with increasing the $n$ number. At $n=4$, the effect of $\Delta \theta$ is small. When the coils are located farther away from the vacuum vessel, the computed plasma response amplitude slowly decays with $r_{c}$. Finally, we point out that the poloidal width of the middle row coils is close to optimum in producing the largest plasma response according to our figures of merit. Therefore, we expect that, with flexible combinations of the middle row and off-middle rows of RMP coils, the present ITER design is capable of providing optimal ELM control capability.

The present investigation is performed for an ITER plasma, for which the coil geometry design has been fixed. Nevertheless the results presented in this work show how close is the ITER coil design to the optimum, according to the plasma response based criteria defined in this work. Moreover, the results should be qualitatively useful for guiding the ELM control coil design in future devices (such as HL-2M currently under construction in China). Obviously, similar investigations can be carried out for a given plasma scenario, on a present day or future device, in order to obtain more quantitative optimization for the RMP coil geometry.

\section{Acknowledgments}

This work is part funded by National Magnetic Confinement Fusion Science Program under grant Nos. 2014GB107004 and 2015GB104004, by National Natural Science Foundation of China 
(NSFC) [grant numbers 11275041 and 11428512] and by the RCUK Energy Programme [grant number EP/I501045]. The views and opinions expressed herein do not necessarily reflect those of the European Commission. L. N. Z. thanks Drs. C. Liu, G. L. Xia, Y. L. He, J. Ren, T. T. Wu and L. B. Li for useful discussions.

\section{References}

[1] Connor J.W. 1998 Plasma Phys. Control. Fusion 40531

[2] Zohm H. 1996 Plasma Phys. Control. Fusion 38105

[3] Loarte A. et al 2007 Nucl. Fusion 47 S203

[4] Evans T.E. et al 2006 Nature Phys. 2419

[5] Liang Y. et al 2007 Phys. Rev. Lett. 98265004

[6] Kirk A. et al 2010 Nucl. Fusion $\mathbf{5 0} 034008$

[7] Suttrop W. et al 2011 Phys. Rev. Lett. 106225004

[8] Jeon Y.M. et al 2012 Phys. Rev. Lett. 109035004

[9] Heyn M.F. et al 2008 Nucl. Fusion 48024005

[10] Becoulet M. et al 2009 Nucl. Fusion 49085011

[11] Liu Y.Q. et al 2010 Phys. Plasmas 17122502

[12] Liu Y.Q. et al 2010 Plasma Phys. Control. Fusion 52045011

[13] Lanctot M.J. et al 2010 Phys. Plasmas 17030701

[14] Park G. et al 2010 Phys. Plasmas 17102503

[15] Lanctot M.J. et al 2011 Phys. Plasmas 18056121

[16] Liu Y.Q. 2011 Nucl. Fusion 51083002

[17] Ferraro N.M. 2012 Phys. Plasmas 19056105

[18] Chapman I.T. et al 2012 Plasma Phys. Control. Fusion 54105013

[19] Orain F. et al 2013 Phys. Plasmas 20102510

[20] Chapman I.T. et al 2014 Nucl. Fusion 55013004

[21] Haskey S.R. et al 2014 Plasma Phys. Control. Fusion 56035005

[22] Wang Z.R. et al 2015 Phys. Rev. Lett. 114145005

[23] Liu Y.Q. et al 2015 Nucl. Fusion 55063027

[24] Haskey S.R. et al 2015 Plasma Phys. Control. Fusion 57025015 
[25] Ryan D.A. et al 2015 Plasma Phys. Control. Fusion 57095008

[26] Schaffer M. et al 2008 Nucl. Fusion 48024004

[27]Liu Y.Q. et al 2012 Plasma Phys. Control. Fusion 54124013

[28] Liu Y.Q. et al 2000 Phys. Plasmas 73681

[29] Casper T. et al 2014 Nucl. Fusion 54013005

[30] Evans T.E. et al 2013 Nucl. Fusion 53093029

[31] Paz-Soldan C. et al 2015 Phys. Rev. Lett. 114105001

[32] Sun Y. et al 2016 "Non-linear transition from mitigation to suppression of edge localized mode with resonant magnetic perturbation in the EAST tokamak", Phys. Rev. Lett. submitted

[33] Crotinger J. et al 1997 Corsica: a comprehensive simulation of toroidal magnetic fusion devices.

Final report to the LDRD program Technical Report (CA, USA: Lawrence Livermore National Lab)

[34] Daly E. et al 2013 Fusion Sci. Technol. 64168

[35] Loarte A. et al 2014 Nucl. Fusion 54033007

[36] Liu Y.Q. et al 2016 Nucl. Fusion 56066001

[37] Kirk A. et al 2015 Nucl. Fusion 55043011 